\title{
COMPARACIONES INTERREGIONALES DE NIVELES DE PRECIOS Y DE CONSUMO REAL PER CAPITA
}

\author{
PEDRo URIBE JR. ${ }^{1}$ \\ El Colegio de México
}

\section{INTRODUCCIÓN}

El interés en estudiar la posibilidad de comparación internacional o interregional de niveles de precios -y la supuestamente implicada de ingresos y consumos reales - ha renacido en fechas recientes, sobre todo a partir del notable esfuerzo de Gilbert y Kravis [7] y en un alto grado ante las necesidades de los estudios de integración económica en diversas zonas del globo. La no escasa literatura en esta materia ${ }^{2}$ ha aportado algunos resultados nuevos bastante interesantes, sobre todo en el área de definición y medición. Por ejemplo, Paige y Bombach [11] y Beckerman [2] han propuesto enfoques distintos a los tradicionales (por ejemplo, el de Gilbert y Kravis) en cuanto a qué magnitudes económicas deban sujetarse a comparación; Paige y Bombach parten de la comparación de valores agregados sectoriales. Otro punto interesante es el llamado "enfoque no monetario" de Niewarowski [10] y Cseh-Szombathy [4], entre otros, muy criticado en algunos círculos (véase el comentario en [2]), pero que no tiene por qué descartarse. El enfoque "no monetario" parte de la idea de formular índices estadísticamente ponderados, de magnitudes que reflejen bienestar (por ejemplo, consumo de energía per capita, número de automóviles, etc.). En el presente artículo se trabaja dentro de los enfoques tradicionales a este respecto, o sea, vía índices "clásicos" de precios y cantidades.

Dentro del área de definición y medición hay otro resultado en extremo importante, que es el uso de métodos de regresión para definir precios de bienes heterogéneos, recientemente propuesto por Kravis y Lipsey [9].

1 Esta investigación es parte del Proyecto ECIEL (Estudios Conjuntos sobre Integración Económica Latinoamericana), a cargo de un grupo de instituciones de investigación de América Latina, que auspician la Fundación Ford y el BID y que coordina The Brookings Institution. El autor agradece los comentarios del Prof. Timothy King, de la Universidad de Cambridge, sobre una versión anterior de este trabajo. Igualmente, la colaboración de Adalberto García Rocha y Raúl de la Peña, del Centro de Estudios Económicos y Demográficos de El Colegio de México, en la programación y el cómputo de los ejemplos numéricos. Finalmente, deseo expresar mi reconocimiento a Petróleos Mexicanos y a la Secretaría de Obras Públicas por haber cedido tiempo máquina sin costo, en sus respectivos centros de cálculo electrónico.

2 Ver [2] para una revisión crítica de publicaciones recientes. 
Sin embargo, no es éste el tipo de problemas que interesa tratar en este artículo, sino problemas que el autor considera de mayor importancia teórica, ${ }^{3}$ quizá el verdadero "fondo" de la comparabilidad de precios e ingresos: dados bienes homogéneos pero patrones de consumo diferentes $i$ son efectivamente comparables los niveles de precios y los ingresos nacionales? En el fondo, se trata de la comparabilidad de funciones utilidad y niveles de satisfacción, problema que no presenta visos de solución teórica, al menos a corto plazo. Las soluciones viables se pueden buscar a través de la teoría de los números índices, cuya conexión con el problema de "utilidad constante", o sea de comparabilidad de niveles de satisfacción, es bastante conocida. La afirmación de Beckerman [2] de que se trata de un problema puramente de teoría de índices resulta entonces un tanto parcial.

En esta área se ha investigado bastante poco; los únicos trabajos relevantes que el autor conoce son el de Van Ijzeren [15] y el de Kloek y Theil [8]. El presente artículo trata de generalizar los resultados de estos últimos. Bajo este enfoque, la comparabilidad de niveles de precios se expresa en términos de la existencia de una escala cardinal de precios, o sea, de números $x_{1}, x_{2}, \ldots$ tales que $x_{a} / x_{b}$ sea un índice de precios del país $a$ con base en el país $b$. Se puede ver de inmediato que esto equivale a que tal índice satisfaga el criterio de circularidad de Fisher.

Wald [16] y Frisch [6] han demostrado la incompatibilidad del criterio de circularidad y otros criterios de Fisher, también muy importantes. ${ }^{4}$ Concretamente, Frisch ha demostrado que el único índice circular, que a su vez es función sólo de precios relativos - y cantidades o pesos- ${ }^{5}$ y satisface el criterio de identidad -el índice de precios de un país con base en sí mismo es idénticamente la unidades un índice geométrico con pesos constantes. Inmediatamente puede pensarse en dos tipos de soluciones: una consistiría en definir pesos constantes que tengan sentido económico -o estadístico, caso en el cual no se andaría lejos de la idea de ponderación (solamente) de Niewarowski- o bien reconocer que la comparabilidad de precios es sólo aproximada; en este caso habría que construir escalas cardinales aproximadas óptimas. Las soluciones del primer tipo son exploradas por el autor en [14]; en el presente artículo interesan las del segundo.

La discusión de los párrafos anteriores se refiere exclusivamente a la comparabilidad de los niveles de precios. La comparabilidad de los ingresos reales -o más bien la posibilidad de definir ingresos reales relativos - no está implicada por la de los niveles de precios, sino que involucra además a los índices de cantidades. Desde el punto de vista de la teoría de los números índices, se depende no sólo del criterio de circularidad, sino del de reversión de factores. Ahora bien,

3 Este planteamiento es válido tanto si las magnitudes a comparar son precios y cantidades, como si se trata de otras, como los índices de Niewarowski y Cseh-Szombathy o de los productos sectoriales.

4 Estas demostraciones han sido revisadas y reformuladas recientemente por Subramanian Swamy [12].

5 Matemáticamente, el indice es una función homogénea de grado cero en los precios; económicamente, esto significa que el índice no incorpora ilusión monetaria en la medición de los precios; ver sección 2. 
Wald [16] ha demostrado que los criterios de circularidad, reversión de factores y proporcionalidad ${ }^{6}$ son incompatibles. Si la comparación se reduce a dos países, el problema de circularidad no existe, y un índice como el "ideal" de Fisher cumple con el propósito de comparar ingresos, puesto que, al satisfacer el criterio de reversión de factores, el ingreso relativo deflacionado es igual al índice de cantidades. Sin embargo, cuando se trata de más de dos países -razón por la cual Gilbert y Kravis [7] se abstuvieron de comparar entre países europeos, dados índices ideales de Fisher de estos países en términos de los Estados Unidos- el problema no parece tener una solución exacta.

Puede de inmediato formularse la siguiente pregunta: Dadas una escala cardinal aproximada óptima de precios $\left\{x_{a}\right\}$ y una escala cardinal aproximada óptima de cantidades $\left\{y_{a}\right\}$, ¿refleja el producto $\left\{x_{a} y_{a}\right\}$ la posición relativa de los países en cuanto a ingreso monetario? En otras palabras ies posible descomponer el ingreso (relativo) monetario en el producto de una escala de precios y una escala de cantidades o "ingreso real"? La respuesta es afirmativa, hasta errores relativamerite pequeños. En concreto, existen índices para los que puede definirse una ecuación algebraica para este error; por desgracia, parece que las expresiones algebraicas de estos errores para los índices más usuales (Paasche, Laspeyres e "ideal" de Fisher) son bastante difíciles de obtener.

La sección 2 plantea la comparabilidad de precios desde el punto de vista de la satisfacción del criterio de circularidad y considera la forma algebraica del "sesgo de circularidad" (circularity gap en el lenguaje de Fisher) para los índices que serán usados en este artículo: índices de Paasche y Laspeyres, el llamado "índice ideal" de Fisher y un índice geométrico propuesto por éste (ver [5], p. 423, índice número 123) y reintroducido recientemente por Theil [13] en el análisis de demanda. Este índice es el usado por Kloek y Theil en [8] y posee propiedades muy interesantes; por ejemplo, en el contexto de series de tiempo, es un índice aproximado local de utilidad constante [13]. En el análisis transversal el significado de esta propiedad es semejante, salvo que la aproximación puede ser bastante más deficiente (ver [8], p. 536). Este índice será llamado "índice de Fisher-Theil". Se encuentra que el sesgo de circularidad del índice de Fisher-Theil es multiplicativo, el del Laspeyres y el del Fisher aproximadamente aditivos y el del Paasche aparentemente multiplicativo; pero la expresión del sesgo del Paasche y del Fisher es algo complicada.

Estos resultados hacen pensar en dos modelos para la búsqueda de escalas aproximadas óptimas en el sentido de los mínimos cuadrados: un modelo con "errores aditivos" y uno con "errores multiplicativos". El segundo, obviamente, debe ser aplicado al Fisher-Theil; el primero al Laspeyres y al Fisher. Ahora bien, de aplicarse el modelo con errores multiplicativos al Paasche, su solución es común al Paasche, al Laspeyres y al Fisher, como se verá más adelante. De esto se

6 Si los precios de un país se multiplican por una constante, sin afectar a los del país base ni a las cantidades, el valor del índice queda multiplicado por dicha constante. 
ocupan las secciones 3 y 4 desde el punto de vista algebraico, y la sección 5 con ejemplos numéricos. La sección 3 obtiene soluciones para el modelo con errores aditivos y la sección 4 generaliza la solución de Kloek y Theil para el modelo con errores multiplicativos, eliminando el requisito, impuesto por estos autores, de que el índice satisfaga el criterio de reversión de base, que no satisfacen el Paasche y el Laspeyres.

La sección 5 presenta ejemplos numéricos; se obtienen escalas aproximadas óptimas de precios respecto a ocho regiones en las que la Oficina de Estudios sobre Proyecciones Agrícolas del Banco de México dividió al país para su encuesta de ingresos y gastos de 1963 [1]. Los datos han sido tomados de esta publicación. El análisis empírico es altamente agregativo: se trabaja con nueve agregados de satisfactores que corresponden al renglón de alimentos y bebidas, de donde los índices y las comparaciones sólo son válidos para este renglón. Los resultados son bastante interesantes: se advierte que el modelo aditivo es muy eficiente para los índices de Paasche, Laspeyres y Fisher, mientras que el multiplicativo sólo lo es para el Fisher-Theil. En cuanto a los datos, un resultado interesante es que el Distrito Federal viene a corresponder aproximadamente a un nivel medio de precios en las ocho regiones. Una buena confirmación empírica de la validez del enfoque de este artículo se tiene en el hecho de que las escalas cardinales de los cuatro índices son sumamente cercanas: aún si se consideran tanto modelos aditivos como multiplicativos, el orden de las regiones es siempre el mismo; numéricamente, la escala con modelo multiplicativo para el Fisher-Theil (o sea la escala de Kloek y Theil) y las escalas con modelo aditivo para el Paasche, el Laspeyres y el "ideal" de Fisher son prácticamente equivalentes.

La sección 6 considera los índices de cantidades y el problema de descomponer el ingreso monetario en el producto de un componente de precios y un componente de "ingreso real" o "cantidades". Se especifican además los sesgos de reversión de factores de los índices de Paasche, Laspeyres y Fisher-Theil y se relaciona, respecto a este último, con el error en la descomposición aludida del ingreso monetario. La sección 7 considera numéricamente estos problemas, con el material de [1]. La semejanza de los cuatro índices y la relativa ineficiencia del modelo multiplicativo para el Paasche, el Laspeyres y el Fisher se pueden ver en forma casi idéntica en el caso de las cantidades. En cuanto a la descomposición del ingreso monetario, se encuentran errores muy pequeños (del orden del $1 \%$ ) en todos los casos. En un futuro, las escalas de precios y cantidades presentadas en este artículo deberán compararse con el enfoque alternativo, o sea con índices "geométricos óptimos" en el sentido de [14].

\section{LAS COMPARACIONES INTERREGIONALES Y EL CRITERIO DE CIRCULARIDAD}

Un índice arbitrario de precios del país $a$ en términos del país $b$ $(a, b=1,2, \ldots, n)$ se denotará por $X_{a b}$; los índices particulares de Paasche y Laspeyres, "ideal" de Fisher y de Fisher-Theil se denotarán respectivamente por $P_{a b}, L_{a b}, F_{a b}$ y $T_{a b}$. Los índices de cantidades se denotarán como sigue : $Y_{a b}$ será un índice arbitrario; los símbolos $Q_{a b}$, 
$C_{a b}, K_{a b}$ y $S_{a b}$ se reservarán para los índices de cantidades de Paasche, Laspeyres, "ideal" de Fisher y Fisher-Theil respectivamente.

Conviene formular los criterios de Fisher que serán de interés especial en este trabajo:

C1. Criterio de identidad: Para todo país $a$ :

$$
X_{a a} \equiv 1
$$

C2. Criterio de proporcionalidad: Sea $X_{a b}$ una función de los precios en $a,\left\{p_{i a}\right\}$ los precios en $b\left\{p_{i b}\right\}$ y "pesos" que los ponderan, $\left\{w_{i a}\right\}$ y $\left\{w_{i b}\right\}$, para $N$ satisfactores:

$$
X_{a b}=f\left(p_{i a}, w_{i a}, p_{i b}, w_{i b}\right) \quad(i=1,2, \ldots, N)
$$

Si $\lambda$ es un número real distinto de cero:

$$
f\left(\lambda p_{i a}, w_{i a}, p_{i b}, w_{i b}\right)=\lambda f\left(p_{i a}, w_{i a}, p_{i b}, w_{i b}\right)
$$

( $\mathrm{Si}$ los precios en un país, relativos a los de otro, crecen todos en una proporción constante, el índice sufre el mismo crecimiento.)

C3. Criterio de homogeneidad: ${ }^{7}$ Si $\lambda_{1}, \lambda_{2}, \ldots, \lambda_{N}$ son números reales distintos de cero, entonces:

$$
\begin{gathered}
f\left(\lambda_{i} p_{i a}, w_{i a}, \lambda_{i} p_{i b}, w_{i b}\right)=f\left(p_{i a}, w_{i a}, p_{i b}, w_{i b}\right) \\
(i=1,2, \ldots, N)
\end{gathered}
$$

(El índice depende sólo de los precios relativos $p_{i a} / p_{i b}$ y por lo tanto es independiente de las unidades de medición, siempre que, para cada satisfactor, se usen las mismas unidades en los dos países.)

Obviamente, si (2.3) no se satisface, la medición del nivel relativo de precios sufre de un fenómeno análogo a lo que se conoce como "ilusión monetaria".

C4. Criterio de reversión de base. Para toda pareja de países $(a, b)$ :

$$
X_{a b}=\frac{1}{X_{b a}}
$$

C5. Criterio de circularidad. Sean $a, b$ y $c$ tres países arbitrarios. Entonces :

$$
X_{a b} X_{b c}=X_{a c}
$$

C6. Criterio de reversión de factores. Sean $X_{a b}$ un índice de precios y $Y_{a b}$ el índice de cantidades que corresponde a $X_{a b}$, o sea:

$$
Y_{a b}=f\left(q_{i a}, w_{i a}, q_{i b}, w_{i b}\right) \quad(i=1,2, \ldots, N)
$$

7 Llamado de "comensurabilidad" por Fisher. El término usado en este artículo refleja la propiedad matemática exacta que Fisher requiere. 
en que $\left\{q_{i a}\right\},\left\{q_{i b}\right\}$ son cantidades en $a$ y en $b$. Entonces:

$$
X_{a b} Y_{a b}=\frac{M_{a}}{M_{b}}
$$

en que $M_{a}$ es el ingreso (consumo) monetario en $a$ y $M_{b}$ es el ingreso (consumo) monetario en $b$.

En particular, si (2.6) se satisface, el ingreso (consumo) relativo deflacionado, $\frac{M_{b}}{M_{b} X_{a b}}$, es un índice de cantidades (consumo o in-
greso real).

Los criterios aludidos no son ni independientes ni compatibles. Por ejemplo, el criterio de reversión de base es consecuencia del de circularidad y el de identidad, si éstos se dan simultáneamente. ${ }^{8} \mathrm{La}$ implicación en el otro sentido es falsa.

Proposición 1: (Frisch [6]) El único indice que satisface simultáneamente (2.1), (2.3) y (2.5) $)^{9}$ es de la forma:

$$
X_{a b}=\prod_{i=1}^{N}\left(\frac{p_{i a}}{p_{i b}}\right)^{\mathcal{w}_{i}}
$$

en que $\mathrm{w}_{1}, \mathrm{w}_{2}, \ldots, \mathrm{w}_{\mathrm{N}}$ son constantes (independientes de los países $\mathrm{a}, \mathrm{b}, \mathrm{c}$, etc.).

Otro resultado importante es el siguiente:

Proposición 2: (Wald [16]) No existe un indice que satisfaga simultáneamente (2.2), (2.5) y (2.6).10

Estes dos resultados son de importancia decisiva en lo que se tratará en este artículo. Se puede ahora abordar el problema de comparación internacional y expresarlo en el contexto de los criterios de Fisher.

Definición. Sea $X_{a b}$ un índice de precios. Los números $\left\{x_{1}, x_{2}, \ldots, x_{n}\right\}$ forman una escala cardinal de precios asociada al índice $X_{a b}$ si (y sólo si) para toda pareja de países $(a, b)$ se tiene que:

$$
\frac{x_{a}}{x_{b}}=X_{a b}
$$$$
(a, b=1,2, \ldots, n)
$$

Definición. Sea $\{a, b, \ldots\}$ una colección de $n$ países. Los niveles de precios de $\{a, b, \ldots\}$ son cardinalmente comparables si existe un índice de precios $X_{a b}$, que posea una escala cardinal asociada.

La comparabilidad cardinal de los niveles de precios significa que es posible afirmar no sólo si un país es o no más "caro" o barato que

8 Si $X_{a b}$ satisface el criterio de circularidad, $X_{a b} X_{b a}=X_{a a}$, y si satisface el de identidad, esto es, la unidad, de donde se desprende (2.4) en forma inmediata.

9 Criterios de identidad, homogeneidad y circularidad.

10 Criterios de proporcionalidad, circularidad y reversión de factores. 
los demás (esto es, ordenarlos en atención a su nivel de precios) sino que, además, qué tanto más caro o barato es, respecto a cada uno de los demás. Evidentemente, éste es el tipo de comparabilidad que interesa en investigaciones como la presente. Es inmediato advertir que la comparabilidad cardinal depende de la estructura de los números índices que se utilicen. En efecto, se tiene:

Proposición 3: Sea $\mathrm{X}_{\mathrm{ab}}$ un indice de precios. Si $\mathrm{n}>2, \mathrm{X}_{a b}$ posee una escala cardinal asociada si (y sólo si) satisface el criterio de circularidad.

Demostración. Es prácticamente evidente. Supóngase que $X_{a b}$ satisface el criterio de circularidad. Selecciónese un país $c$ arbitrario (pero fijo en todo este razonamiento) y defínanse: $x_{a}=X_{a c}, x_{b}=X_{b c}$, etc. Por (2.5):

$$
x_{a}=X_{a c}=X_{a b} X_{b c}=X_{a b} x_{b}
$$

de donde (2.8) se concluye de inmediato.

Por otra parte, supóngase que existe una escala cardinal de precios asociada con algún índice $X_{a b}$. Entonces:

$$
X_{a b} X_{b c}=\left(\frac{x_{a}}{x_{b}}\right)\left(\frac{x_{b}}{x_{c}}\right)=\frac{x_{a}}{x_{c}}=X_{a c}
$$

o sea, la relación $(2.5)$.

Ahora bien, si $n=2$, basta con los criterios de identidad y reversión de base, puesto que se puede hacer $x_{b}=1, x_{a}=X_{a b}$.

Se concluye de las proposiciones 1 y 3 que solamente para un índice geométrico con pesos constantes existe la posibilidad de comparación cardinal de niveles de precios para tres o más países, si se desea conservar los criterios de homogeneidad e identidad. Es importante conservar estos dos criterios, por razones bastante obvias.

Se advierten entonces dos caminos hacia la solución del problema: o bien se definen pesos constantes, $w_{1}, w_{2}, \ldots, w_{N}$ con sentido económico o estadístico, o bien se busca una escala cardinal aproximada, óptima en algún sentido concreto. La definición de pesos constantes estadísticamente óptimos es abordada por el autor en un trabajo a ser presentado próximamente [14], en el que se introduce el concepto de "índice geométrico óptimo" para precios y para cantidades. Kloek y Theil [8] han encontrado la escala cardinal aproximada óptima ante los siguientes supuestos:

1) (2.8) se satisface con un error multiplicativo;

2) el índice utilizado satisface los criterios de identidad, homogeneidad y reversión de base.

En este artículo se consideran, por una parte, un modelo con errores aditivos, bajo el supuesto de que el índice utilizado satisfaga los criterios de identidad y homogeneidad, y un modelo con errores 
multiplicativos, en que también se elimina el supuesto de que el índice satisfaga el criterio de reversión de base (por ejemplo, el Paasche y el Laspeyres no lo satisfacen). Antes de estudiar estos dos modelos y sus soluciones, conviene analizar la forma algebraica del posible error para cada uno de los índices manejados en este trabajo. En primer lugar, considérese el índice de Fisher-Theil:

$$
T_{a b}=\prod_{i=1}^{N}\left(\frac{p_{i a}}{p_{i b}}\right)^{\frac{w_{i a}+w_{i b}}{2}}
$$

en que $w_{i a}$ es la participación del satisfactor $i$ en el consumo per capita del país $a$. Se tiene:

$$
\begin{aligned}
& T_{a b} T_{b c}=\prod_{i=\mathrm{T}}^{N}\left(\frac{p_{i a}}{p_{i b}}\right)^{\frac{w_{i a}+w_{i b}}{2}}\left(\frac{p_{i b}}{p_{i c}}\right)^{\frac{w_{i b}+w_{i c}}{2}} \\
& =T_{a c}\left(\prod_{i=1}^{N}\left(p_{i a}\right) \frac{w_{i b}-w_{i c}}{2}\left(p_{i b}\right) \frac{w_{i c}-w_{i a}}{2}\left(p_{i c}\right) \frac{w_{i a}-w_{i b}}{2}\right)
\end{aligned}
$$

Para $c$ fijo, $T_{a b} T_{b c}$ se puede escribir como:

$$
T_{a b} T_{b c}=T_{a c} v_{a b}
$$

o sea, el "sesgo de circularidad" puede representarse mediante un modelo multiplicativo. Puede verse que si las estructuras presupuestales (medidas por las participaciones $w_{i a}, w_{i b}$, etc.) no son muy distintas, los exponentes en la última expresión en (2.10) son cercanos a cero, de donde, en tal caso, el criterio de circularidad se satisface en forma aproximada.

Para estudiar el sesgo de circularidad de los índices de Paasche y Laspeyres y del índice "ideal" de Fisher, conviene usar notación matricial. El vector columna ${ }^{11} p_{a}$ tendrá $N$ elementos, $p_{1 a}, \ldots, p_{N a}$, los precios de los $N$ satisfactores en el país a (análogamente, se usará más adelante el vector de cantidades $q_{a}$ ); el vector columna $w_{a}$ tendrá como elementos las $N$ participaciones presupuestales en el país $a$.

El índice de precios de Paasche, ordinariamente escrito como:

$$
P_{a b}=\frac{\sum_{i=1}^{N} p_{i a} q_{i a}}{\sum_{i=1}^{N} p_{i b} q_{i a}}=\frac{1}{\sum_{i=1}^{N} w_{i a} \frac{p_{i b}}{p_{i a}}}
$$

11 Todos los vectores serán columnas; los renglones serán columnas traspuestas y la trasposición se denotará por medio de apóstrofes. El vector ı (iota) será un vector cuyos elementos son todos iguales a la unidad. El acento circunflejo convertirá vectores en matrices diagonales: $\widehat{x}$ es la matriz de elementos $x_{i} \delta_{i j}$, en que $\delta_{i j}$ es la delta de Kronecker. 

puede escribirse:

$$
P_{a b}=\frac{1}{w_{a}^{\prime} \hat{p}_{a}^{-1} p_{b}}
$$

Se obtiene entonces:

$$
\begin{aligned}
\left(P_{a b} P_{b c}\right)^{-1} & =w_{a}^{\prime} \hat{p}_{a}^{-1}\left(\frac{p_{b} q_{b}^{\prime}}{M_{b}}\right) p_{c} \\
& =P_{a c}{ }^{-1}-w_{a}^{\prime} \widehat{p}_{a}^{-1}\left(I-\frac{p_{b} q_{b}^{\prime}}{M_{b}}\right) p_{c}
\end{aligned}
$$

de donde:

$$
P_{a b} P_{b c}=\frac{P_{a c}}{1-P_{a c} w_{a}^{\prime} \hat{p}_{a}^{-1}\left(I-\frac{p_{b} q_{b}^{\prime}}{M_{b}}\right) p_{c}}
$$

Para el índice de Laspeyres se tiene:

$$
L_{a b}=\sum_{i=1}^{N} w_{i b} \frac{p_{i a}}{p_{i b}}=w_{b}^{\prime} \hat{p}_{b}^{-1} p_{a}
$$

de donde:

$$
\begin{aligned}
L_{a b} L_{b c} & =w_{c}^{\prime} \hat{p}_{c}^{-1}\left(\frac{p_{b} q_{b}^{\prime}}{M_{b}}\right) p_{a} \\
& =L_{a c}-w^{\prime}{ }_{c} \hat{p}_{c}^{-1}\left(I-\frac{p_{b} q_{b}^{\prime}}{M_{b}}\right) p_{a}
\end{aligned}
$$

Finalmente, para el índice "ideal" de Fisher:

$$
F_{a b}=\left(P_{a b} L_{a b}\right)^{1 / 2}=\left(\frac{w_{b}^{\prime} \widehat{p}_{b}^{-1} p_{a}}{w_{a}^{\prime} \hat{p}_{a}^{-1} p_{b}}\right)^{1 / 2}
$$

de donde:

$$
\text { (2.16) } \begin{aligned}
F_{a b} F_{b c} & =\frac{F_{a c}}{\left(1-P_{a c} w_{a}^{\prime} \hat{p}_{a}^{-1}\left(I-\frac{p_{b} q_{b}^{\prime}}{M_{b}}\right) p_{c}\right)^{1 / 2}} \\
& +\left(\frac{w_{c}^{\prime} \hat{p}_{c}^{-1}\left(I-\frac{p_{b} q_{b}^{\prime}}{M_{b}}\right) p_{a} P_{a c}}{1-P_{a c} w_{a}^{\prime} \hat{p}_{a}^{-1}\left(I-\frac{p_{b} q_{b}^{\prime}}{M_{b}}\right) p_{c}}\right)^{1 / 2}
\end{aligned}
$$


Aparentemente, sólo para el caso del índice de Laspeyres está más o menos claro que el sesgo de circularidad es aproximadamente aditivo. Las expresiones para los índices de Paasche y Fisher son notablemente más complicadas; particularmente el sesgo del índice "ideal" de Fisher presenta una mezcla de una parte aditiva y una parte aparentemente multiplicativa.

Como consecuencia, en este trabajo se proponen dos modelos para la aproximación de la ecuación (2.8):

Modelo I: (Errores Multiplicativos).

$$
X_{a b}=\frac{x_{a}}{x_{b}} v_{a b}^{\prime}
$$

que se puede escribir, en forma aditiva:

$$
\log X_{a b}=\log x_{a}-\log x_{b}+v_{a b}
$$

en que $v_{a b}=\log v_{a b}^{\prime}$. Este es, esencialmente, el modelo usado por Kloek $\mathrm{y}$ Theil.

Modelo II: (Errores Aditivos).

$$
X_{a b} x_{b}=x_{a}+u_{a b}
$$

Empíricamente, los modelos serán aplicados: el modelo I a los cuatro índices; el modelo II a los índices de Paasche, Laspeyres e "ideal" de Fisher, puesto que el sesgo del Fisher-Theil es claramente multiplicativo. Se podría eliminar del modelo I el índice de Laspeyres, pero se verá más adelante que la solución del modelo I aplicando cualquiera de los tres, Laspeyres, Paasche e "ideal" de Fisher, es la misma, o sea, el modelo resulta ser común a los tres índices.

Debe notarse que la ecuación (2.8) se satisface si $x_{a}$ y $x_{b}$ se multiplican ambos por una misma constante, de donde toda escala cardinal está definida hasta una constante multiplicativa, o sea, hasta elección de unidades. En consecuencia, en la búsqueda de la escala cardinal aproximada óptima para ambos modelos citados arriba existe un grado de libertad. La optimización debe, pues, llevarse a cabo bajo una restricción que especifique el valor numérico de alguna función escalar (por ejemplo: media aritmética, magnitud, etc.) del vector que representa a la escala, o sea, el vector de componentes $x_{a}, x_{b}, \ldots$, etc.

\section{ESCALAS APROXIMADAS PARA EL MODELO CON ERRORES ADITIVOS}

En notación matricial, (2.19) puede escribirse como sigue (ver nota 11 para aclaraciones respecto a notación):

$$
X \widehat{x}=x \iota^{\prime}+U
$$

Una escala aproximada óptima minimiza la distancia entre $X \hat{x}$ y $x \iota^{\prime}$. Resulta claro que cualquier distancia podría ser una función- 
criterio. Por ejemplo, es tentador considerar una forma cuadrática positiva definida

$$
d\left(X \widehat{x}, x \iota^{\prime}\right)=\left(\operatorname{tr} U^{\prime} H U\right)^{1 / 2}
$$

en que el operador tr denota la traza de una matriz y $H$ es una matriz positiva definida y simétrica. Si $H$ no es la identidad, (3.2) corresponde a la idea de ponderar en forma distinta los errores cometidos al comparar unos países con otros. Parece que este camino no añade mucha generalidad y sí algo más de complejidad al problema. En este trabajo, $H$ es la identidad y por lo tanto se considera la minimización del cuadrado de la distancia euclideana:

$$
\begin{aligned}
\operatorname{tr} U^{\prime} U & =\operatorname{tr}\left(X \hat{x}-x \iota^{\prime}\right)^{\prime}\left(X \hat{x}-x \iota^{\prime}\right) \\
& =\operatorname{tr} \hat{x} X^{\prime} X \hat{x}-2 x^{\prime} X x+n x^{\prime} x \\
& =x^{\prime} \hat{r} x-2 x^{\prime} X x+n x^{\prime} x
\end{aligned}
$$

en que $r_{b}=\sum_{a=1}^{n} X_{a b}^{2}$, como puede comprobarse directamente. Esto no es sino la suma de cuadrados de los "errores" $u_{a b}$, de modo que el criterio (3.3) corresponde al criterio de mínimos cuadrados.

Se minimizará (3.3) bajo dos condiciones laterales alternativas: una sobre la magnitud de $x$, la otra sobre el valor medio de sus elementos. Obviamente la primera es una restricción sobre el "cuadrado medio" de los elementos de $x$. Es claro que si, además, se desea imponer condiciones de no negatividad de la solución en $x$, el problema se convierte en uno de programación cuadrática. En este artículo se trabajará con métodos lagrangianos ordinarios; en algunos casos es posible asertar la no negatividad de la solución (por ejemplo, acudiendo al teorema de Perron y Frobenius sobre matrices no negativas; ver [3], p. 278).

Considérese en primer lugar la minimización de (3.3) bajo la condición:

$$
x^{\prime} x=A
$$

en que $A$ es un número no negativo dado de antemano. La función lagrangiana correspondiente es:

$$
\varphi(x, \lambda)=x^{\prime}(\hat{r}-2 X-(n-\lambda) I) x-\lambda A
$$

de modo que:

$$
-1 / 2 \frac{\delta \varphi(x, \lambda)}{\delta x}=\left(X+X^{\prime}-\hat{r}-\lambda_{1} I\right) x=0
$$

en que $\lambda_{1}=n-\lambda$. La ecuación (3.5) establece que $x$ es un vector característico de la matriz (simétrica) $X+X^{\prime}-\widehat{r}$, asociado a la raíz (real) $\lambda_{1}$, aún desconocida. Se demuestra en seguida que $\lambda_{1}$ es la máxima raíz característica de $X+X^{\prime}-\widehat{r}$. 
Considérese el cociente de Rayleigh para la forma cuadrática $x^{\prime}\left(X+X^{\prime}-\hat{r}\right) x$ :

$$
R(x)=\frac{x^{\prime}\left(X+X^{\prime}-\hat{r}\right) x}{x^{\prime} x}
$$

Evidentemente, $R(x)$ es invariante ante la multiplicación de $x$ por un escalar cualquiera, o sea, ante cambios en la magnitud de $x$. Concretamente, supóngase que $x^{\prime} x=A$. Se tiene entonces:

$$
\begin{aligned}
A R(x) & =x^{\prime}\left(X+X^{\prime}-\hat{r}\right) x \\
& =n A-\operatorname{tr} U^{\prime} U
\end{aligned}
$$

de modo que, si $x$ minimiza $\operatorname{tr} U^{\prime} U$ en la esfera $x^{\prime} x=A$, entonces maximiza $A R(x)$ en la misma esfera. Por el teorema del min-max de Courant y Fischer ${ }^{12}$ el máximo de $R(x)$ en la esfera $x^{\prime} x=1$ es:

$$
\max R(x)=\lambda_{0} \dot{=} R\left(x^{0}\right)
$$

en que $\lambda_{0}$ es la máxima raíz característica de $X+X^{\prime}-\widehat{r}$ y $x^{0}$ su vector característico unitario correspondiente. Obviamente, el máximo en la esfera $x^{\prime} x=A$ es $A \lambda_{0}$ y corresponde a $x^{0} \sqrt{A}$ como vector característico.

Se concluye que $\lambda_{1}=\lambda_{0}$ y el vector solución es $x^{0} \sqrt{A}$. Si la matriz diagonal $\hat{r}$ tiene todos sus elementos menores que los elementos diagonales de $X+X^{\prime}$, o sea, si:

$$
1 / 2 \sum_{a=1}^{n} X_{a b}^{2}<X_{b b} \quad(b=1,2, \ldots, n)
$$

$\lambda_{1}$ es simple y positiva, por el teorema de Perron y Frobenius; ${ }^{13} x$ es además único hasta una constante multiplicativa y sus elementos son estrictamente positivos. Desde luego, si (3.6) no se cumple, $\lambda_{1}$ puede ser múltiple, y entonces la solución en $x$ es cualquier elemento (de magnitud $A$ ) del espacio generado por los vectores correspondientes a $\lambda_{1}$; la dimensión de este espacio es igual a la multiplicidad de $\lambda_{1}$.

La segunda condición de normalización se refiere al valor medio de la escala cardinal, o sea:

$$
\frac{\iota^{\prime} x}{n}=\bar{x}
$$

en que $\bar{x}$ es un escalar dado. Sea $n \bar{x}=B$ y considérese la función lagrangiana: 


$$
\varphi(x, \lambda)=x^{\prime}(\hat{r}-2 X+n I) x-2 \lambda\left(B-\imath^{\prime} x\right)
$$

Se tiene entonces:

$$
-1 / 2 \frac{\partial \Psi}{\partial x}=\left(X+X^{\prime}-\hat{r}-n I\right) x-\lambda_{\iota}=0
$$

así que, si $X+X^{\prime}-\hat{r}-n I$ no es singular:

$$
x=\lambda\left(X+X^{\prime}-\widehat{r}-n I\right)^{-1} \iota
$$

Se advierte de inmediato que:

$$
\lambda=\frac{B}{\iota^{\prime}\left(X+X^{\prime}-\widehat{r}-n I\right)^{-1} \iota}
$$

4. UNA GENERALIZACIÓN DEL MODELO CON ERRORES MULTIPLICATIVOS DE KLOEK Y THEIL

En esta sección se considera el modelo (2.18). Sean $Z_{t i b}=\log X_{a b}$, $z_{a}=\log x_{a}$. Se tiene:

$$
Z=z \imath^{\prime}-\imath z^{\prime}+V
$$

en que $V=\left(v_{a b}\right)$. Kloek y Theil [8] utilizan la condición $\imath^{\prime} z=0$, que es equivalente a la condición de que la media geométrica $\left(\underset{a}{\prod_{a}} x_{a}\right)^{1 / n}$ sea la unidad. Considérese la minimización de:

$$
\begin{aligned}
\operatorname{tr} V^{\prime} V & =\operatorname{tr} Z^{\prime} Z-2 \iota^{\prime} Z z-2 z^{\prime} Z \iota \\
& +2 n z^{\prime} z-2\left(\iota^{\prime} z\right)^{2}
\end{aligned}
$$

bajo la condición $\iota^{\prime} z=0$. La función lagrangiana correspondiente es:

$$
\begin{aligned}
\psi(z, \mu) & =\operatorname{tr} Z^{\prime} Z-2 \iota^{\prime} Z z-2 z^{\prime} Z \iota \\
& +2 n z^{\prime} z-2\left(\iota^{\prime} z\right)^{2}-4 \mu \iota^{\prime} z \\
& =\operatorname{tr} Z^{\prime} Z-4 \iota^{\prime} S(Z) z+2 n z^{\prime} z \\
& -2\left(\iota^{\prime} z\right)^{2}-4 \mu \iota^{\prime} z
\end{aligned}
$$

en que $S(Z)=1 / 2\left(Z-Z^{\prime}\right)$ es una matriz antisimétrica. Antes de proceder a la minimización, es conveniente considerar qué son los elementos de $S(Z)$ en términos de los índices de precios.

Defínase el reverso ${ }^{14}$ del índice $X_{a b}$ como:

$$
\operatorname{rev}\left(X_{a b}\right)=1 / X_{b a}
$$

14 Esta terminología es de Frisch [6]. 
Evidentemente, $X_{a b}$ satisface el criterio de reversión de base si (y sólo si) es idéntico a su reverso. El índice "ideal" de Fisher y el índice de Fisher-Theil satisfacen este criterio. Para los índices de Paasche y Laspeyres se tiene:

$$
\begin{aligned}
& \operatorname{rev}\left(P_{a b}\right)=L_{a b} \\
& \operatorname{rev}\left(L_{a b}\right)=P_{a b}
\end{aligned}
$$

Ahora bien, los elementos de $S(Z)$ son:

$$
\begin{aligned}
S(Z)_{a, b} & =\frac{\log X_{a b}-\log X_{b a}}{2} \\
& =\log \left(\frac{X_{a b}}{X_{b a}}\right)^{1 / 2} \\
& =\log \sqrt{X_{a b} \operatorname{rev}\left(X_{a b}\right)}
\end{aligned}
$$

Consecuentemente, si el índice $X_{a b}$ satisface el criterio de reversión de base, $S(Z)=Z$. En el caso concreto de los índices de Paasche, Laspeyres y Fisher, $S(Z)$ consiste precisamente de los logaritmos de los índices "ideales" de Fisher. La solución al problema de mínimo es entonces la misma, en este modelo, para los índices de Paasche, Laspeyres e "ideal" de Fisher.

Considérese ahora el problema de minimización. Se tiene, volviendo a (4.3):

$$
\frac{1}{4} \frac{\partial \psi}{\partial z}=-S(Z)+n z-\left(\iota^{\prime} z\right)-\mu \iota=0
$$

o sea :

$$
z=\frac{S(Z) \iota+\mu \iota}{n}
$$

en donde se ha hecho uso de la restricción ${ }^{\prime} z=0$ y del hecho de que $-S(Z)=S^{\prime}(Z)$. Como $\iota^{\prime} z=0$, se tiene, de (4.4):

$$
0=\iota^{\prime} z=\frac{\iota^{\prime} S(Z) \iota}{n}+\mu
$$

y, como $S(Z)$ es antisimétrica $\left(\iota^{\prime} S(Z) \iota=0\right), \mu$ es necesariamente cero. Se concluye que:

$$
z=\frac{S(Z) \iota}{n}
$$

o sea :

$$
x_{a}=\left(\prod_{b=1}^{n} \sqrt{X_{a b} \operatorname{rev}\left(X_{a b}\right)}\right)^{1 / n}
$$


En el caso especial en que $X_{a b}$ satisfaga el criterio de reversión de base:

$$
x_{a}=\left(\prod_{b=1}^{n} X_{a b}\right)^{1 / n}
$$

la media geométrica de $X_{a b}$ (sobre $b$ ), que es el resultado de Kloek y Theil. Para el Paasche, el Laspeyres y el Fisher, la solución es la media geométrica (sobre $b$ ) de los índices ideales de Fisher, $F_{a b}$.

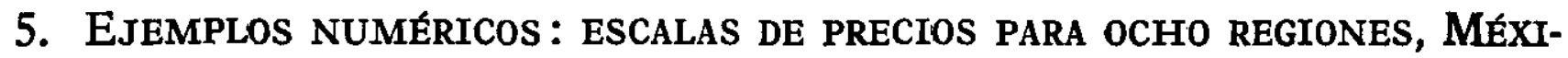
Co, 1963

Se han utilizado los datos de la encuesta de ingresos y gastos familiares realizada en 1963 por la Oficina de Estudios sobre Proyecciones Agrícolas del Banco de México [1], para obtener índices de precios de ocho regiones en que se dividió al país en dicha encuesta. Las regiones y los Estados que comprenden son los siguientes:

Región 1. Pacífico Norte: Baja California (Norte y Sur) y Sonora. Región 2. Norte: Chihuahua, Coahuila, Nuevo León y Tamaulipas. Región 3. Golfo-Sureste: Campeche, Quintana Roo, Tabasco, Veracruz y Yucatán.

Región 4. Pacífico-Centro: Durango, Nayarit, Sinaloa y Zacatecas.

\section{Cuadro 1}

MÉxico: Prectos POR KILOGRAMO Y PARTICIPACION EN EL CONSUMO DE ALIMENTOS Y BEBIDAS DE NUEVE AGREGADOS DE SATISFACTORES EN OCHO REGIONES, 1963

\begin{tabular}{|c|c|c|c|c|c|c|c|c|}
\hline$\underbrace{\text { Bención }}_{\text {Lquegado }}$ & $\begin{array}{l}\text { 3. Peorftion } \\
\text { Morte }\end{array}$ & 2. Norto & 3.. Golfo & 4. Paoffico & 5. Deffo & 6. Contro & 7. Mistrito & $\begin{array}{l}\text { 8. Paoffe100 } \\
\text { Sur }\end{array}$ \\
\hline 1. xarl J tos & $(2.2475=1$ & (.2854) & $\begin{array}{l}1.2152 \\
(1091)\end{array}$ & ' 1.0892 & $\left(\begin{array}{l}1.1211 \\
(1762)\end{array}\right.$ & $\begin{array}{l}1.0293 \\
(1526)\end{array}$ & $\begin{array}{l}1.0937 \\
(.0502)\end{array}$ & $\begin{array}{l}1.0988 \\
(1840)\end{array}$ \\
\hline $\begin{array}{l}\text { 2. } 2 \times 180 \text { J was } \\
\text { production }\end{array}$ & $(.0866)$ & $\begin{array}{l}2.9754 \\
(2.1067)\end{array}$ & $\begin{array}{l}3.4882 \\
(0788)\end{array}$ & $\begin{array}{l}3.1746 \\
(0763)\end{array}$ & $\begin{array}{c}3.2131 \\
(2029)\end{array}$ & $\begin{array}{r}3.4346 \\
(1120)\end{array}$ & $\begin{array}{l}2.9873 \\
(1143)\end{array}$ & $\begin{array}{r}3.2889 \\
(0926) \\
\end{array}$ \\
\hline $\begin{array}{l}\text { 3. Noular, is } \\
\text { guinosias, } \\
\text { cereales }\end{array}$ & $\begin{array}{l}2.7840 \\
(.1099)\end{array}$ & $\begin{array}{l}2.4186 \\
(.1129)\end{array}$ & $\begin{array}{l}2.8446 \\
(.0974)\end{array}$ & $\left(\begin{array}{l}2.2611 \\
(.1417)\end{array}\right.$ & $\begin{array}{l}2.4701 \\
(.1333) \\
\end{array}$ & $\begin{array}{r}2.6609 \\
(.1320) \\
\end{array}$ & $\begin{array}{l}2.4811 \\
(.0696)\end{array}$ & $\begin{array}{r}2.3551 \\
(.1143)\end{array}$ \\
\hline 4. Arioneses & $\begin{array}{l}1.9362 \\
(.0285)\end{array}$ & $\begin{array}{r}1.7783 \\
(.0329)\end{array}$ & $\begin{array}{l}1.7953 \\
(.0272)\end{array}$ & $\begin{array}{l}1.8130 \\
(0.49)\end{array}$ & $\begin{array}{l}2.8104 \\
(.0336)\end{array}$ & $\begin{array}{l}1.7216 \\
(.0353)\end{array}$ & $\begin{array}{l}1.7551 \\
(.0283) \\
\end{array}$ & $\begin{array}{r}2.6953 \\
(.0 \times 23)\end{array}$ \\
\hline $\begin{array}{l}\text { 3. Vexduran } \\
\text { erates }\end{array}$ & $\begin{array}{l}2.9111 \\
(21235)\end{array}$ & $\left(\begin{array}{l}2.2831 \\
(0904)\end{array}\right.$ & $\begin{array}{l}1.6949 \\
(60785)\end{array}$ & $\begin{array}{l}1.7220 \\
(.0613)\end{array}$ & $\begin{array}{l}1.7699 \\
(.0823)\end{array}$ & $\begin{array}{l}1.6771 \\
(.0906)\end{array}$ & $\begin{array}{r}1.8162 \\
(.1210)\end{array}$ & $\begin{array}{l}1.6727 \\
60049\end{array}$ \\
\hline 6. Orwase y & $\begin{array}{l}6.6056 \\
(0827)\end{array}$ & $\begin{array}{l}6.9725 \\
(.0903)\end{array}$ & $\begin{array}{l}8.5259 \\
(.0598)\end{array}$ & $\begin{array}{l}8.3051 \\
(1027)\end{array}$ & $\begin{array}{l}6.9268 \\
(.0843)\end{array}$ & $\begin{array}{r}7.4172 \\
(0678)\end{array}$ & $\begin{array}{l}6.4001 \\
6.0629)\end{array}$ & $\begin{array}{r}1.9378 \\
(.0278)\end{array}$ \\
\hline 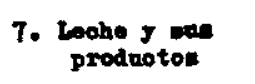 & $\begin{array}{l}2.7602 \\
(.1215)\end{array}$ & $\begin{array}{l}1.5266 \\
(2.1028)\end{array}$ & $\begin{array}{l}2.3575 \\
(.0947) \\
\end{array}$ & $\begin{array}{l}1.3220 \\
(1095)\end{array}$ & $\begin{array}{r}1.2167 \\
(.1262\end{array}$ & $\begin{array}{l}1.2037 \\
(1079)\end{array}$ & $\begin{array}{l}1.2778 \\
(1195)\end{array}$ & $\begin{array}{l}1.3869 \\
(.0070)\end{array}$ \\
\hline 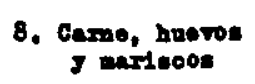 & $\begin{array}{l}11.8724 \\
(.3228)\end{array}$ & $\begin{array}{l}10.6619 \\
(2849)\end{array}$ & $\begin{array}{l}23.0087 \\
(.3741\end{array}$ & $\begin{array}{l}9.3573 \\
(1896)\end{array}$ & $\begin{array}{l}9.9454 \\
(2130)\end{array}$ & $\begin{array}{r}9.3730 \\
(247)\end{array}$ & $\begin{array}{l}21.6062 \\
(3757)\end{array}$ & $\begin{array}{l}10.6234 \\
(.2755)\end{array}$ \\
\hline $\begin{array}{l}\text { 9. Bebidan J } \\
\text { otros }\end{array}$ & $\left(\begin{array}{c}7.4012 \\
(.0811)\end{array}\right.$ & $\begin{array}{l}8.4404 \\
60742\end{array}$ & $\left(\begin{array}{c}7.7828 \\
(.0710)\end{array}\right.$ & $(.0749)$ & $\left(\begin{array}{l}6.0183 \\
(0482)\end{array}\right.$ & $\left.\begin{array}{l}4.6257 \\
60500\end{array}\right]$ & $\begin{array}{l}9.1536 \\
(.0591)\end{array}$ & $\begin{array}{l}5.0042 \\
(1027)\end{array}$ \\
\hline
\end{tabular}

Fuente: Referencia 1.

a Las cifras entre paréntesis son las participaciones en el gasto total familiar en alimentos $\mathrm{y}$ bebidas. 
Región 5. Bajío: Aguascalientes, Colima, Guanajuato, Jalisco, Querétaro y San Luis Potosí.

Región 6. Centro: Hidalgo, México, Michoacán, Morelos, Puebla y Tlaxcala.

Región 7. Distrito Federal.

Región 8. Pacífico Sur: Chiapas, Guerrero y Oaxaca.

Los precios y participaciones presupuestales utilizados en el cálculo de los índices se refieren a nueve agregados de satisfactores, dentro del renglón de alimentos y bebidas solamente; en el cuadro 1 se dan los precios por kilogramo y las participaciones presupuestales de cada agregado, para cada región.

En primer lugar se consideran los resultados del modelo con errores multiplicativos. Para el índice de Fisher-Theil el modelo resulta bastante eficiente en cuanto a circularidad, o sea en cuanto a la cercanía de $T_{a b}$ respecto al cociente $x_{a} / x_{b}$ de los elementos de la escala cardinal óptima; ver cuadro 3 . Los índices de Paasche, Laspeyres y Fisher son notablemente menos cercanos al cociente $x_{a} / x_{b}$, al menos para este modelo. El cuadro 2, a continuación, presenta las escalas cardinales aproximadas óptimas para los cuatro índices.

\section{Cuadro 2}

ESCALAS CARDINALES APROXIMADAS ÓPTIMAS : MODELO CON ERRORES MULTIPLICATIVOS

Escalas óptimas

Región

Indices: Paasche, Laspeyres $e$ "ideal" de Fisher

1. Pacífico Norte

2. Norte

3. Golfo-Sureste

4. Pacífico-Centro

5. Bajío

6. Centro

7. Distrito Federal

8. Pacífico Sur

\subsection{2}

1.0208

1.0274

1.0085

1.0056

0.9994

1.0152

0.9981
Fisher-Theil

Número de orden

El rango de variación de la escala para el índice de Fisher-Thiel es bastante más amplio que el de la escala para los otros tres índices; aparentemente, esta última es un tanto "insensible", en el sentido de que las variaciones del cociente $x_{a} / x_{b}$ (sobre $a$ y $b$ ) son menores que las de $P_{a b}, L_{a b}$ y $F_{a b}$; ver cuadro 4 .

El cuadro 5 presenta seis escalas de niveles de precios, que corresponden al modelo con errores aditivos. Las tres primeras escalas provienen de restringir la magnitud del vector-escala, o sea, se trata del primer vector característico de $X+X^{\prime}-\widehat{r}$ bajo la condición de que $x^{\prime} x=8$, o sea, $\sqrt{\frac{x^{\prime} x}{n}}=1$. Las tres siguientes provienen de res- 
Cuadro 3

CiRCularidad DEL ÍNDICE DE FISHER-THEIL:

$T_{a b}$ Y RELACIÓN $x_{a} / x_{b}$ (entre paréntesis)

\begin{tabular}{|c|c|c|c|c|c|c|c|c|}
\hline 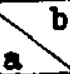 & 1 & 2 & 3 & 4 & 5 & 6 & 7 & 8 \\
\hline 1 & 1 & $\begin{array}{r}1.1532 \\
(1.1670) \\
\end{array}$ & $\begin{array}{c}1.0875 \\
(1.0996) \\
\end{array}$ & $\begin{array}{c}1.2871 \\
(1.2713) \\
\end{array}$ & $\begin{array}{c}1.3039 \\
(1.2871) \\
\end{array}$ & $\begin{array}{r}1.3332 \\
(1.3275) \\
\end{array}$ & & $\begin{array}{r}1.3878 \\
(1.3701) \\
\end{array}$ \\
\hline 2 & $\begin{array}{l}.8672 \\
(.8569)\end{array}$ & 1 & $\begin{array}{l}.9371 \\
.9423) \\
\end{array}$ & $\begin{array}{c}1.0858 \\
(1.0894)\end{array}$ & $\begin{array}{r}1.0992 \\
(1.1030) \\
\end{array}$ & $\begin{array}{c}1.1307 \\
(1.1376)\end{array}$ & $\begin{array}{c}1.0276 \\
(1.0305)\end{array}$ & $\begin{array}{r}1.1853 \\
(1.1740)\end{array}$ \\
\hline 3 & $\begin{array}{l}.9195 \\
(.9094)\end{array}$ & $\begin{array}{c}1.0672 \\
(1.0612)\end{array}$ & 1 & $\begin{array}{r}1.1576 \\
(1.1561) \\
\end{array}$ & $\begin{array}{c}1.1661 \\
(1.1705)\end{array}$ & $\begin{array}{c}1.2072 \\
(1.2073)\end{array}$ & $\begin{array}{c}1.0898 \\
(1.0936)\end{array}$ & $\begin{array}{c}1.2333 \\
(1.2460) \\
\end{array}$ \\
\hline 4 & $\begin{array}{l}.7769 \\
(.7866) \\
\end{array}$ & $\begin{array}{l}.9210 \\
(.9179 \\
\end{array}$ & $\begin{array}{l}.8639 \\
.8649) \\
\end{array}$ & 1 & $\begin{array}{r}1.0111 \\
(1.0124) \\
\end{array}$ & $\begin{array}{c}1.0369 \\
(1.0442)\end{array}$ & $\begin{array}{l}.9482 \\
(.9459) \\
\end{array}$ & $\begin{array}{r}1.0936 \\
(1.0777)\end{array}$ \\
\hline 5 & $\begin{array}{l}.7661 \\
(.7769)\end{array}$ & $\begin{array}{c}.9098 \\
(.9066 \\
\end{array}$ & $\begin{array}{r}.8576 \\
(.8543) \\
\end{array}$ & $\begin{array}{l}.9890 \\
(.9877) \\
\end{array}$ & 1 & $\begin{array}{c}1.0267 \\
(1.0314)\end{array}$ & $\begin{array}{r}.9421 \\
(.9343) \\
\end{array}$ & $\begin{array}{r}1.0653 \\
(1.0644)\end{array}$ \\
\hline 6 & $\begin{array}{l}.7501 \\
(.7533 \\
\end{array}$ & $\begin{array}{l}.8544 \\
(.0791 \\
\end{array}$ & $\begin{array}{l}.8284 \\
.8283 \\
\end{array}$ & $\begin{array}{r}.9627 \\
(.9277) \\
\end{array}$ & $\begin{array}{l}.9740 \\
(.9696 \\
\end{array}$ & 1 & $\begin{array}{c}.9108 \\
(.2059) \\
\end{array}$ & $\begin{array}{r}1.0141 \\
(1.0320) \\
\end{array}$ \\
\hline 7 & $\begin{array}{r}.6482 \\
.8316 \\
\end{array}$ & $\left.\begin{array}{r}.9731 \\
.9704\end{array}\right)$ & $\begin{array}{r}.9176 \\
.9144 \\
\end{array}$ & $\begin{array}{r}1.0547 \\
(1.0572) \\
\end{array}$ & $\begin{array}{c}1.0614 \\
(1.0703)\end{array}$ & $\begin{array}{c}1.0980 \\
(1.1039)\end{array}$ & 1 & $\begin{array}{r}1.1280 \\
(1.1393)\end{array}$ \\
\hline 8 & $\begin{array}{l}.7206 \\
(.7300\end{array}$ & $\begin{array}{r}.8436 \\
(.8518 \\
\end{array}$ & $\begin{array}{r}.8109 \\
(.8026)\end{array}$ & $\begin{array}{r}.9144 \\
(.9279) \\
\end{array}$ & $\begin{array}{r}.9387 \\
. .9395\end{array}$ & $\begin{array}{r}.9861 \\
(.9689) \\
\end{array}$ & $\begin{array}{l}.8865 \\
(.8777)\end{array}$ & 1 \\
\hline
\end{tabular}

Cuadro 4

Circularidad de los Índices de PaAsche, Laspeyres e "ideal" de Fisher: ÍNDICES $\mathrm{P}_{\mathrm{ab}}, \mathrm{L}_{\mathrm{ab}}, \mathrm{F}_{a b}$ Y REJACIÓN $\mathrm{X}_{\mathrm{a}} / \mathrm{x}_{\mathrm{b}}$ (entre paréntesis)

MODELOS CON ERRORES MULTIPLICATIVOS

\begin{tabular}{|c|c|c|c|c|c|c|c|c|}
\hline 20 & 1 & 2 & 3 & 4 & 5 & 6 & 7 & 8 \\
\hline$I$ & 1 & $\begin{array}{r}1.1202 \\
1.1931 \\
1.1561 \\
(1.0229)\end{array}$ & $\begin{array}{l}1.0272 \\
1.1590 \\
1.0911 \\
(1.0164)\end{array}$ & $\begin{array}{r}1.1982 \\
1.3949 \\
1.2928 \\
(1.0354)\end{array}$ & $\begin{array}{r}1.2189 \\
1.4134 \\
1.3125 \\
(1.0384)\end{array}$ & $\begin{array}{c}1.2444 \\
1.4417 \\
1.3394 \\
(1.0448)\end{array}$ & $\begin{array}{r}1.1263 \\
1.2454 \\
1.1844 \\
(1.0286)\end{array}$ & $\begin{array}{l}1.3081 \\
1.4479 \\
1.3763 \\
(1.0462) \\
\end{array}$ \\
\hline 2 & $\begin{array}{r}.8381 \\
.8927 \\
.8650 \\
(.9776) \\
\end{array}$ & 1 & $\begin{array}{r}.9302 \\
.9447 \\
.9374 \\
(.9236)\end{array}$ & $\begin{array}{l}1.0794 \\
1.0916 \\
1.0855\end{array}$ & $\begin{array}{l}2.0918 \\
1.2061 \\
1.0990\end{array}$ & $\begin{array}{l}1.1139 \\
1.1466 \\
1.1301\end{array}$ & $\begin{array}{l}1.0245 \\
1.0318 \\
1.0281\end{array}$ & $\begin{array}{l}1.1607 \\
1.1844 \\
1.1725\end{array}$ \\
\hline 3 & $\begin{array}{l}.8628 \\
.9735 \\
.9165 \\
.9832)\end{array}$ & $\begin{array}{l}1.0585 \\
1.0751 \\
1.0668 \\
(\underline{1.0065)}\end{array}$ & 1 & $\begin{array}{l}1.1689 \\
1.1437 \\
1.1562 \\
(1.0187)\end{array}$ & $\begin{array}{l}1.1775 \\
1.1537 \\
1.1656 \\
(\underline{1.0217)}\end{array}$ & $\begin{array}{r}1.2183 \\
1.1953 \\
1.2067 \\
(\underline{1.0280})\end{array}$ & $\begin{array}{r}1.0873 \\
1.0920 \\
1.0897 \\
(1.0120)\end{array}$ & $\begin{array}{r}1.2195 \\
1.2294 \\
1.2245 \\
(1.0294)\end{array}$ \\
\hline 4 & $\begin{array}{r}.7169 \\
.8346 \\
.7735 \\
(.9658)\end{array}$ & $\begin{array}{r}.9161 \\
.9265 \\
.9213 \\
(.9880) \\
\end{array}$ & $\begin{array}{r}.8743 \\
.8555 \\
.8649 \\
(.9816) \\
\end{array}$ & $I$ & $\begin{array}{r}1.0107 \\
1.0108 \\
1.0107 \\
(1.0029)\end{array}$ & $\begin{array}{r}1.0367 \\
1.0397 \\
1.0382 \\
(1.0091)\end{array}$ & $\begin{array}{r}.9599 \\
.9374 \\
.9486 \\
(.9934) \\
\end{array}$ & $\begin{array}{l}1.0749 \\
1.0775 \\
1.0762 \\
(\underline{1.0104)}\end{array}$ \\
\hline 5 & $\begin{array}{r}.6936 \\
.8204 \\
.7629 \\
. .9630 \\
\end{array}$ & $\begin{array}{r}.9040 \\
.9159 \\
.9100 \\
.9851 \\
\end{array}$ & $\begin{array}{r}.8668 \\
.8492 \\
.8579 \\
(.9788) \\
\end{array}$ & $\begin{array}{r}.9893 \\
.9895 \\
.9894 \\
(.9972) \\
\end{array}$ & 1 & $\begin{array}{r}1.0224 \\
3.0312 \\
2.0268 \\
(1.0062)\end{array}$ & $\begin{array}{r}.9516 \\
.9328 \\
.9422 \\
(.9905) \\
\end{array}$ & $\begin{array}{l}2.0533 \\
1.0532 \\
1.0532 \\
(1.0075)\end{array}$ \\
\hline 6 & $\begin{array}{r}.6936 \\
.8036 \\
.7466 \\
.92711)\end{array}$ & $\begin{array}{r}.8721 \\
.8978 \\
.8249 \\
.2790 \\
\end{array}$ & $\begin{array}{r}.8366 \\
.8202 \\
.8287 \\
(.2727) \\
\end{array}$ & $\begin{array}{r}.9638 \\
.9646 \\
.9632 \\
.9210 \\
\end{array}$ & $\begin{array}{r}.9697 \\
.9781 \\
.9739 \\
(.9938)\end{array}$ & 1 & $\begin{array}{r}.9095 \\
.9107 \\
.9101 \\
. .9844) \\
\end{array}$ & $\begin{array}{l}1.0068 \\
1.0067 \\
1.0668 \\
(1.0013)\end{array}$ \\
\hline 7 & $\begin{array}{r}.8029 \\
.8878 \\
.8443 \\
(.2722)\end{array}$ & $\begin{array}{r}.9692 \\
.9761 \\
.9726 \\
(.9245)\end{array}$ & $\begin{array}{r}.9157 \\
.9197 \\
.9177 \\
(.9881) \\
\end{array}$ & $\begin{array}{r}1.0668 \\
1.0418 \\
1.0542 \\
(1.0066)\end{array}$ & $\begin{array}{r}1.0721 \\
1.0508 \\
1.0614 \\
(1.0095)\end{array}$ & $\begin{array}{r}1.0981 \\
1.0995 \\
1.0988 \\
(1.0158)\end{array}$ & 1 & $\begin{array}{l}1.1035 \\
1.1495 \\
1.1263 \\
(1.0171)\end{array}$ \\
\hline 8 & $\begin{array}{l}.6906 \\
.7645 \\
.7266 \\
.9559)\end{array}$ & $\begin{array}{r}.8443 \\
.8615 \\
.8529 \\
(.9778) \\
\end{array}$ & $\begin{array}{r}.8134 \\
.8200 \\
.8167 \\
(.2715) \\
\end{array}$ & $\begin{array}{r}.9281 \\
.9303 \\
.9292 \\
(.2897) \\
\end{array}$ & $\begin{array}{r}.9495 \\
.9494 \\
.9495 \\
(.9925) \\
\end{array}$ & $\begin{array}{r}.9933 \\
.9933 \\
.9933 \\
(.9987)\end{array}$ & $\begin{array}{r}.8700 \\
.9062 \\
.8879 \\
(.983 I) \\
\end{array}$ & 1 \\
\hline
\end{tabular}


Cuadro 5

EsCALAS ÓPTTMAS DE PRECIOS PARA MODELOS CON ERROYES ADITIVOS

\begin{tabular}{|c|c|c|c|c|c|c|c|}
\hline \multirow{2}{*}{ Regtón } & \multirow{2}{*}{$\begin{array}{l}\text { Túnero } \\
\text { de } \\
\text { oxden }\end{array}$} & \multicolumn{3}{|c|}{ Magnitud restringida } & \multicolumn{3}{|c|}{ Nedia restringida } \\
\hline & & Paasohe & Laspeyres & Fisher & Paasche & Laspeyres & Fisher \\
\hline 1. Paoífico Norte & 1 & 1.1861 & 1.2117 & 1.1998 & 1.1915 & 1.2183 & 1.2061 \\
\hline 2. Norte & 3 & 1.0262 & 1.0250 & 1.0253 & 1.0309 & 1.0307 & 1.0304 \\
\hline 3. Golfo Sureste & 2 & 1.0902 & 1.0869 & 1.0881 & 1.0952 & 1.0929 & 1.0937 \\
\hline 4. Pacífico Centro & 5 & .9427 & .9365 & .9396 & .9471 & .9417 & .9444 \\
\hline 5. Bajío & 6 & .9316 & .9251 & .9286 & .9359 & .9303 & .9332 \\
\hline 6. Contro. & 7 & .9043 & .8977 & .9011 & .9086 & .9027 & .9057 \\
\hline 7. Distrito Pederal & 4 & .9978 & .9950 & .9962 & 1.0025 & 1.0005 & 1.0012 \\
\hline 8. Paolfico Sur & 8 & .8841 & .8779 & .8808 & .8883 & .8828 & .8854 \\
\hline
\end{tabular}

Cuadro 6

Circularidad del fNDICE DE PaAsche bajo el MODELO aditivo: $P_{a b}$ Y RELACIONES $x_{a} / x_{b}$

\begin{tabular}{|c|c|c|c|c|c|c|c|c|}
\hline & 1 & 2 & 3 & 4 & 5 & 6 & 7 & 8 \\
\hline 1 & 1 & $\begin{array}{l}1.1202 \\
1.1558 \\
1.1558\end{array}$ & $\begin{array}{l}1.0272 \\
1.0880 \\
1.0879\end{array}$ & $\begin{array}{l}1.1981 \\
1.2582 \\
1.2581\end{array}$ & $\begin{array}{l}1.2189 \\
1.2732 \\
1.2731\end{array}$ & $\begin{array}{l}1.2444 \\
1.3116 \\
1.3114\end{array}$ & $\begin{array}{l}1.1263 \\
1.1887 \\
1.1385\end{array}$ & $\begin{array}{l}1.3081 \\
1.3416 \\
1.3413\end{array}$ \\
\hline 2 & $\begin{array}{l}.8381 \\
.8652 \\
.8652\end{array}$ & 1 & $\begin{array}{r}.9302 \\
.9413 \\
.9413\end{array}$ & $\begin{array}{l}1.0794 \\
1.0886 \\
1.0885\end{array}$ & $\begin{array}{l}1.0918 \\
1.1015 \\
1.1015\end{array}$ & $\begin{array}{l}1.1339 \\
1.1348 \\
1.1346\end{array}$ & $\begin{array}{l}1.0245 \\
1.0285 \\
1.0283\end{array}$ & $\begin{array}{l}1.1607 \\
1.1607 \\
1.1605\end{array}$ \\
\hline 3 & $\begin{array}{r}.8628 \\
.9191 \\
.9192\end{array}$ & $\begin{array}{l}1.0585 \\
1.0624 \\
1.0624\end{array}$ & 1 & $\begin{array}{r}1.1689 \\
-1.1565 \\
1.1564\end{array}$ & $\begin{array}{l}1.1775 \\
1.1702 \\
1.1702\end{array}$ & $\begin{array}{l}1.2183 \\
1.2056 \\
1.2054\end{array}$ & $\begin{array}{l}1.0873 \\
1.0926 \\
1.0925\end{array}$ & $\begin{array}{l}1.2195 \\
1.2331 \\
1.2329\end{array}$ \\
\hline 4 & $\begin{array}{l}.7169 \\
.7948 \\
.7949\end{array}$ & $\begin{array}{r}.9161 \\
.9186 \\
.9187\end{array}$ & $\begin{array}{l}.8743 \\
.3647 \\
.8648\end{array}$ & 1 & $\begin{array}{l}1.0107 \\
1.0119 \\
1.0120\end{array}$ & $\begin{array}{l}1.0367 \\
1.0425 \\
1.0424\end{array}$ & $\begin{array}{l}.9599 \\
.9448 \\
.9447\end{array}$ & $\begin{array}{l}1.0749 \\
1.0663 \\
1.0662\end{array}$ \\
\hline 5 & $\begin{array}{l}.7075 \\
.7854 \\
.7855\end{array}$ & $\begin{array}{l}.9040 \\
.9078 \\
.9078\end{array}$ & $\begin{array}{l}.8664 \\
.8545 \\
.8545\end{array}$ & $\begin{array}{l}.9893 \\
.9882 \\
.9882\end{array}$ & 1 & $\begin{array}{l}1.0224 \\
1.0302 \\
1.0300\end{array}$ & $\begin{array}{l}.9516 \\
.9337 \\
.9336\end{array}$ & $\begin{array}{l}1.0533 \\
1.0537 \\
1.0536\end{array}$ \\
\hline 6 & $\begin{array}{l}.6936 \\
.7624 \\
.7626\end{array}$ & $\begin{array}{l}.8721 \\
.8812 \\
.8814\end{array}$ & $\begin{array}{l}.8366 \\
.8295 \\
.8296\end{array}$ & $\begin{array}{l}.9618 \\
.9593 \\
.9593\end{array}$ & $\begin{array}{l}.9697 \\
.9707 \\
.9708\end{array}$ & 1 & $\begin{array}{l}.9095 \\
.9063 \\
.9063\end{array}$ & $\begin{array}{l}1.0068 \\
1.0228 \\
1.0229\end{array}$ \\
\hline 7 & $\begin{array}{l}.8029 \\
.8412 \\
.8414\end{array}$ & $\begin{array}{l}.9692 \\
.9723 \\
.9725\end{array}$ & $\begin{array}{r}.9157 \\
.9152 \\
.9154\end{array}$ & $\begin{array}{l}1.0668 \\
1.0584 \\
1.0585\end{array}$ & $\begin{array}{l}1.0721 \\
1.0711 \\
1.0712\end{array}$ & $\begin{array}{l}1.0981 \\
1.1034 \\
1.1033\end{array}$ & 1 & $\begin{array}{l}1.1035 \\
1.1286 \\
1.1286\end{array}$ \\
\hline 8 & $\begin{array}{l}.6906 \\
.7454 \\
.7455\end{array}$ & $\begin{array}{l}.8443 \\
.8615 \\
.8617\end{array}$ & $\begin{array}{l}.8134 \\
.8110 \\
.8111\end{array}$ & $\begin{array}{l}.9281 \\
.9378 \\
.9379\end{array}$ & $\begin{array}{l}.9495 \\
.9490 \\
.9491\end{array}$ & $\begin{array}{r}.9933 \\
.9777 \\
.9777\end{array}$ & $\begin{array}{l}.8700 \\
.8860 \\
.8861\end{array}$ & 1 \\
\hline
\end{tabular}


tringir la media aritmética del vector-escala, o sea, se trata de números proporcionales a la media aritmética de los renglones de $\left(X+X^{\prime}-\right.$ $\widehat{r}-n \mathrm{I})^{-1}$, bajo la condición $\imath^{\prime} x=8$, o sea, $\bar{x}=1$.

Resalta desde luego la total coincidencia de este resultado con el ordenamiento obtenido a partir del modelo con errores multiplicativos. Por otra parte, hay una gran coincidencia numérica con la escala proveniente del índice de Fisher-Theil, no así con la escala obtenida a partir de los otros tres índices en el modelo con errores multiplicativos. A partir de los cuadros 6, 7 y 8, se advierte la mayor eficiencia (en cuanto a circularidad) de las escalas derivadas del modelo con errores aditivos para los índices de Paasche, Laspeyres y Fisher, comparable a la eficiencia del modelo multiplicativo para el Fisher-Theil; Ia primera cifra en cada casilla en cada cuadro, es el índice correspondiente $\left(P_{a b}, L_{a b}\right.$ y $F_{a b}$ respectivamente); la segunda es la relación $x_{a} / x_{b}$ para la escala con magnitud restringida, y la tercera es la relación $x_{a} / x_{b}$ para el modelo con media restringida.

\section{Cuadro 7}

CIRCULARIDAD DEL INDICE DE LASPEYRES BAJO EL MODELO ADITTVO: $L_{a b} Y$ RELACTONES $x_{a} / x_{b}$

\begin{tabular}{|c|c|c|c|c|c|c|c|c|}
\hline $\mathrm{b}$ & 1 & 2 & 3 & $4:$ & 5 & 6 & 7 & 8 \\
\hline 1 & 1 & $\begin{array}{l}1.1931 \\
1.1821 \\
1.1820\end{array}$ & $\begin{array}{l}1.1590 \\
1.1148 \\
1.1147\end{array}$ & $\begin{array}{l}1.3949 \\
1.2939 \\
1.2937\end{array}$ & $\begin{array}{l}1.4134 \\
1.3097 \\
1.3096\end{array}$ & $\begin{array}{l}1.4417 \\
1.3497 \\
1.3496\end{array}$ & $\begin{array}{l}1.2454 \\
1.2177 \\
1.2177\end{array}$ & $\begin{array}{l}1.4479 \\
1.3802 \\
1.3800\end{array}$ \\
\hline 2 & $\begin{array}{l}.8927 \\
.8459 \\
.8460\end{array}$ & 1 & $\begin{array}{l}.9447 \\
.9430 \\
.9431\end{array}$ & $\begin{array}{r}1.0916 \\
1.0945 \\
1.0945\end{array}$ & $\begin{array}{l}1.1061 \\
1.1079 \\
1.1079\end{array}$ & $\begin{array}{l}1.1466 \\
1.1418 \\
1.1418\end{array}$ & $\begin{array}{l}1.0318 \\
1.0301 \\
1.0302\end{array}$ & $\begin{array}{l}1.1844 \\
1.1675 \\
1.1675\end{array}$ \\
\hline 3 & $\begin{array}{l}.9735 \\
.8971 \\
.8971\end{array}$ & $\begin{array}{l}1.0751 \\
1.0604 \\
1.0603\end{array}$ & 1 & $\begin{array}{l}1.1437 \\
1.1607 \\
1.1606\end{array}$ & $\begin{array}{l}1.1537 \\
1.1749 \\
1.1747\end{array}$ & $\begin{array}{l}1.1953 \\
1.2108 \\
1.2107\end{array}$ & $\begin{array}{l}1.0920 \\
1.0924 \\
1.0924\end{array}$ & $\begin{array}{l}1.2294 \\
1.2381 \\
1.2380\end{array}$ \\
\hline 4 & $\begin{array}{l}.8346 \\
.7729 \\
.7730\end{array}$ & $\begin{array}{l}.9265 \\
.9136 \\
.9137\end{array}$ & $\begin{array}{l}.8555 \\
.8616 \\
.8617\end{array}$ & 1 & $\begin{array}{l}1.0108 \\
1.0122 \\
1.0123\end{array}$ & $\begin{array}{l}1.0397 \\
1.0432 \\
1.0432\end{array}$ & $\begin{array}{r}.9374 \\
.9412 \\
.9412\end{array}$ & $\begin{array}{l}1.0775 \\
1.0667 \\
1.0667\end{array}$ \\
\hline 5 & $\begin{array}{l}.8204 \\
.7636 \\
.7634\end{array}$ & $\begin{array}{l}.9159 \\
.9026 \\
.9026\end{array}$ & $\begin{array}{l}.8492 \\
.8512 \\
.8512\end{array}$ & $\begin{array}{l}.9895 \\
.9879 \\
.9880\end{array}$ & 1 & $\begin{array}{l}1.0312 \\
1.0306 \\
1.0306\end{array}$ & $\begin{array}{r}.9328 \\
.9298 \\
.9298\end{array}$ & $\begin{array}{l}1.0532 \\
1.0538 \\
1.0538\end{array}$ \\
\hline 6 & $\begin{array}{l}.8036 \\
.7409 \\
.7409\end{array}$ & $\begin{array}{r}.8970 \\
.8758 \\
.8758\end{array}$ & $\begin{array}{l}.8208 \\
.8259 \\
.8260\end{array}$ & $\begin{array}{r}.9646 \\
.9586 \\
.9586\end{array}$ & $\begin{array}{l}.9781 \\
.9703 \\
.9703\end{array}$ & 1 & $\begin{array}{l}.9107 \\
.9022 \\
.9022\end{array}$ & $\begin{array}{l}1.0067 \\
1.0226 \\
1.0225\end{array}$ \\
\hline$\dot{\gamma}$ & $\begin{array}{l}.8878 \\
.8212 \\
.8212\end{array}$ & $\begin{array}{r}.9761 \\
.9708 \\
.9707\end{array}$ & $\begin{array}{l}.9197 \\
.9154 \\
.9155\end{array}$ & $\begin{array}{l}1.0418 \\
1.0625 \\
1.0624\end{array}$ & $\begin{array}{l}1.0508 \\
1.0755 \\
1.0755\end{array}$ & $\begin{array}{l}1.0995 \\
1.1084 \\
1.1083\end{array}$ & 1 & $\begin{array}{l}1.1495 \\
1.1334 \\
1.1333\end{array}$ \\
\hline 8 & $\begin{array}{l}.7645 \\
.7246 \\
.7246\end{array}$ & $\begin{array}{l}.8615 \\
.8565 \\
.8565\end{array}$ & $\begin{array}{l}.8200 \\
.8077 \\
.8078\end{array}$ & $\begin{array}{r}.9303 \\
.9375 \\
.9375\end{array}$ & $\begin{array}{l}.9494 \\
.9489 \\
.9489\end{array}$ & $\begin{array}{l}.9933 \\
.9779 \\
.9780\end{array}$ & $\begin{array}{r}.9062 \\
.8823 \\
.8824\end{array}$ & 1 \\
\hline
\end{tabular}




\section{Cuadro 8}

Circularidad del ÍNDice "IDEAL" DE Fisher Bajo el Modelo aditivo: $F_{a b} Y$ RELACIONES $x_{a} / b$

\begin{tabular}{|c|c|c|c|c|c|c|c|c|}
\hline 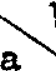 & 1 & 2 & 3 & 4 & 5 & 6 & 7 & 8 \\
\hline 1 & 1 & $\begin{array}{l}1.1561 \\
1.1702 \\
1.1705\end{array}$ & $\begin{array}{l}1.0911 \\
1.1027 \\
1.1028\end{array}$ & $\begin{array}{l}1.2928 \\
1.2769 \\
1.2771\end{array}$ & $\begin{array}{l}1.3125 \\
1.2921 \\
1.2924\end{array}$ & $\begin{array}{l}1.3394 \\
1.3315 \\
1.3317\end{array}$ & $\begin{array}{l}1.1844 \\
1.2044 \\
1.2047\end{array}$ & $\begin{array}{l}1.3763 \\
1.3622 \\
1 \div 3622\end{array}$ \\
\hline 2 & $\begin{array}{l}.8650 \\
.8545 \\
.8543\end{array}$ & 1 & $\begin{array}{l}.9374 \\
.9423 \\
.9421\end{array}$ & $\begin{array}{l}1.0855 \\
1.0912 \\
1.0911\end{array}$ & $\begin{array}{l}1.0990 \\
1.1042 \\
1.1042\end{array}$ & $\begin{array}{l}1.1301 \\
1.1378 \\
1.1377\end{array}$ & $\begin{array}{l}1.0281 \\
1.0292 \\
1.0292\end{array}$ & $\begin{array}{l}1.1725 \\
1.1641 \\
1.1638\end{array}$ \\
\hline 3 & $\begin{array}{l}.9165 \\
.9069 \\
.9068\end{array}$ & $\begin{array}{l}1.0668 \\
1.0612 \\
1.0614\end{array}$ & 1 & $\begin{array}{l}1.1562 \\
1.1580 \\
1.1581\end{array}$ & $\begin{array}{l}1.1656 \\
1.1718 \\
1.1720\end{array}$ & $\begin{array}{l}1.2067 \\
1.2075 \\
1.2076\end{array}$ & $\begin{array}{l}1.0897 \\
1.0923 \\
1.0924\end{array}$ & $\begin{array}{l}1.2245 \\
1.2354 \\
1.2353\end{array}$ \\
\hline 4 & $\begin{array}{l}.7735 \\
.7831 \\
.7830\end{array}$ & $\begin{array}{r}.9213 \\
.9164 \\
.9165\end{array}$ & $\begin{array}{l}.8649 \\
.8635 \\
.8635\end{array}$ & 1 & $\begin{array}{r}1.0107 \\
.1 .0119 \\
1.0120\end{array}$ & $\begin{array}{l}1.0382 \\
1.0427 \\
1.0427\end{array}$ & $\begin{array}{l}.9486 \\
.9432 \\
.9433\end{array}$ & $\begin{array}{l}1.0762 \\
1.0668 \\
1.0667\end{array}$ \\
\hline 5 & $\begin{array}{r}.7619 \\
.7739 \\
.7737\end{array}$ & $\begin{array}{l}.9100 \\
.9057 \\
.9057\end{array}$ & $\begin{array}{l}.8579 \\
.8534 \\
.8533\end{array}$ & $\begin{array}{r}.9894 \\
.9883 \\
.9881\end{array}$ & 1 & $\begin{array}{l}1.0268 \\
1.0304 \\
1.0304\end{array}$ & $\begin{array}{l}.9422 \\
.9321 \\
.9321\end{array}$ & $\begin{array}{l}1.0532 \\
1.0543 \\
1.0540\end{array}$ \\
\hline 6 & $\begin{array}{l}.7466 \\
.7511 \\
.7509\end{array}$ & $\begin{array}{l}.8849 \\
.8789 \\
.3790\end{array}$ & $\begin{array}{l}.8287 \\
.8282 \\
.8281\end{array}$ & $\begin{array}{r}.9632 \\
.9591 \\
.9590\end{array}$ & $\begin{array}{l}.9739 \\
.9705 \\
.9705\end{array}$ & 1 & $\begin{array}{r}.9101 \\
.9046 \\
.9046\end{array}$ & $\begin{array}{l}1.0668 \\
1.0231 \\
1.0229\end{array}$ \\
\hline 7 & $\begin{array}{l}.8443 \\
.8303 \\
.8302\end{array}$ & $\begin{array}{l}.9726 \\
.9716 \\
.9717\end{array}$ & $\begin{array}{r}.9177 \\
.9155 \\
.9154\end{array}$ & $\begin{array}{l}1.0542 \\
1.0602 \\
1.0601\end{array}$ & $\begin{array}{l}1.0614 \\
1.0728 \\
1.0729\end{array}$ & $\begin{array}{l}1.0988 \\
1.1055 \\
1.1054\end{array}$ & 1 & $\begin{array}{l}1.1263 \\
1.1310 \\
1.1308\end{array}$ \\
\hline 8 & $\begin{array}{l}.7266 \\
.7341 \\
.7341\end{array}$ & $\begin{array}{l}.8529 \\
.8590 \\
.8593\end{array}$ & $\begin{array}{l}.8167 \\
.8095 \\
.8095\end{array}$ & $\begin{array}{l}.9292 \\
.9374 \\
.9375\end{array}$ & $\begin{array}{l}.9495 \\
.9485 \\
.9488\end{array}$ & $\begin{array}{l}.9933 \\
.9774 \\
.9776\end{array}$ & $\begin{array}{l}.8879 \\
.8842 \\
.8843\end{array}$ & 1 \\
\hline
\end{tabular}

Empíricamente, los resultados son interesantes. La región en la que la alimentación es más cara es la Pacífico Norte, lo cual no debe extrañar. Lo que llama la atención en cambio, es que la segunda región más cara sea la Golfo-Sureste, tradicionalmente considerada como una región "barata". Una explicación puede radicar en que, habiendo una proporción no despreciable de autoconsumo en el régimen de vida de los habitantes de esta zona, los productos disponibles en el mercado no reflejan el "verdadero" costo de la alimentación y ésta es "cara" para quien viene de fuera, que compra en el mercado. Probablemente esta explicación es errónea; por una parte, la zona de mayor autoconsumo, según quienes "conocen" la economía regional del país, es la que aquí se define como "Pacífico Sur", que resulta ser la más barata. La relación entre autoconsumo y "carestía" de la economía de mercado es bastante nebulosa. Por otra parte, los resultados en cuanto a los índices de cantidades parecen contradecir la conjetura del volumen de autoconsumo: la región Golfo-Sureste es la más alta en cuanto 
a la escala de cantidades. El autor se inclina a pensar en que, si los "conocedores" tienen razón, la explicación radica en defectos de los datos, quizá concretamente en defectos de la muestra del Banco de México, sesgada en esa región hacia los ingresos altos. Por otra parte, es interesante advertir que el Distrito Federal viene a representar aproximadamente la media de nivel de costo de la alimentación del país, cuando menos a este nivel de agregación.

\section{Escalas DE CANTIDADES Y NIVELES DE INGRESO REAL}

En forma enteramente análoga a los índices de precios, es posible definir escalas cardinales aproximadas óptimas para los índices de cantidades; éstas aparecen, para las ocho regiones de la sección 5, al principio de la sección 7 . El problema crucial de la definición del nivel de ingreso real es la relación entre los índices de cantidades y los índices de precios y, en el enfoque dado en este artículo, entre las escalas cardinales de precios y las escalas cardinales de cantidades.

Concretamente, en el caso de dos países, el ingreso (o consumo) monetario relativo deflacionado con el índice de precios:

$$
\frac{M_{a}}{M_{b} X_{a b}}
$$

es un índice de cantidades si $X_{a b}$ y el índice de cantidades $Y_{a b}$, satisfacen el criterio de reversión de factores. En caso contrario, el significado de la relación (6.1) no es algo muy claro; aun más, no puede representar una relación de "consumo real", puesto que no es un "consumo físico relativo" como el índice de cantidades.

Los índices de Paasche y Laspeyres no satisfacen el criterio de rever. sión de factores; en efecto:

$$
\begin{aligned}
P_{a b} Q_{a b} & =\left(w_{a}^{\prime} \widehat{p}_{a}^{-1} p_{b} w_{a}^{\prime} \widehat{q}_{a}^{-1} q_{b}\right)^{-1} \\
& =\left(w_{a}^{\prime} \widehat{p}^{-1} p_{b} q_{b}^{\prime} \widehat{q}_{a}^{-1} w_{a}\right)^{-1} \\
& =\left(q_{a}^{\prime} p_{b} q_{b}^{\prime} p_{a}\right)^{-1} M_{a}^{2} \\
& =\frac{M_{a}^{2}}{M_{a b} M_{b a}}
\end{aligned}
$$

en que $M_{a b}$ es el gasto total cuando las cantidades consumidas en $a$ se compran a los precios de $b$.

Para el Laspeyres se obtiene:

$$
\begin{aligned}
L_{a b} C_{a b} & =w_{b}^{\prime} \hat{p}_{b}^{-1} p_{a} q_{a}^{\prime} \widehat{q}_{b}^{-1} w_{b} \\
& =\frac{M_{a b} M_{b a}}{M^{2}{ }_{b}}
\end{aligned}
$$


Comparando (6.2) y (6.3) se nota que el índice "ideal". de Fisher sí satisface el criterio de reversión de factores. Además, es fácil ver que :

$$
P_{a b} C_{a b}=L_{a b} Q_{a b}=\frac{M_{a}}{M_{b}}
$$

Para el Fisher-Theil se tiene:

$$
\begin{gathered}
\text { (6.5) } T_{a b} S_{a b}=\prod_{i=1}^{N}\left(\frac{p_{i a} q_{i a}}{p_{i b} q_{i b}}\right)^{\frac{w_{i a} w_{i b}}{2}}=\frac{M_{a}}{M_{b}} \exp \Sigma \frac{w_{i a} w_{i b}}{2} \ldots \\
\ldots \log \frac{w_{i a}}{w_{i b}}=\frac{M_{a}}{M_{b}} \exp \frac{1}{2}\left(\sum_{i=1}^{N} w_{i a} \log \frac{w_{i a}}{w_{i b}} \ldots\right. \\
\left.\ldots-\sum_{i=1}^{N} w_{i b} \log \frac{w_{i b}}{w_{i a}}\right)=\frac{M_{a}}{M_{b}} \exp \frac{1}{2} \Delta I_{a b}
\end{gathered}
$$

La cantidad entre paréntesis en la penúltima expresión en (6.5) tiene una interpretación interesante en términos de la teoría de la información: se trata de la diferencia de información entre dos experimentos: en uno, el investigador tiene información a priori consistente en la distribución presupuestal del país $b$ (los números $w_{i b}$ ); el experimento cambia esta información a la distribución presupuestal ( $a$ posteriori) del país $a$. En el segundo experimento, la información $a$ priori es la distribución presupuestal de $a$ y la $a$ posteriori es la de $b$. Se concluye que si las distribuciones presupuestales de ambos países no son muy diferentes, este término es pequeño y $T_{a b} S_{a b}$ es aproximadamente igual a $M_{a} / M_{b}$.

De existir un índice que satisficiera el criterio de circularidad y el de reversión de factores a la vez, se tendría:

$$
X_{a b} Y_{a b}=\frac{x_{a}}{x_{b}} \frac{y_{a}}{y_{b}}=\frac{M_{a}}{M_{b}}
$$

de modo que, si $M_{a}, M_{b}$, etc., se escalan para representar una escala de gastos monetarios relativos:

$$
\frac{x_{a} y_{a}}{\mathrm{x}_{b} y_{b}}=\frac{M_{a} / M}{M_{b} / M}\left(M=1 / n \underset{a}{\Sigma} M_{a} \text { ó } M=\left(\prod_{a} M_{a}\right)^{1 / n} \text { etc. }\right)
$$

o sea, $x_{a} y_{a}, x_{b}, y_{b}, \ldots$, etc., formarían una escala de gastos monetarios relativos, cuyos componentes son, por una parte el nivel relativo de precios $x_{a}$ y por otra el nivel relativo de "consumo físico", o sea la escala de cantidades $y_{a}$.

Tal representación es imposible en forma exacta, por el teorema de Wald (proposición 2, sección 2 de este artículo). Sin embargo, es posible llegar a representaciones aproximadas. En particular, considérense las escalas cardinales de Kloek y Theil para precios y cantidades, con el índice de Fisher-Theil: 


$$
\begin{aligned}
x_{a} y_{a} & =\prod_{b=1}^{n}\left(T_{a b} S_{a b}\right)^{1 / n}=\frac{M_{a} \exp \frac{1}{2 n} \sum_{b=1}^{n} \Delta I_{a b}}{M .} \\
& =\frac{M_{a}}{M .} \exp \frac{1}{2} \Delta I_{a} .
\end{aligned}
$$

en que $M$. es la media geométrica $\operatorname{II}_{b=1}^{n} M_{b}$ y $\Delta I_{a}$. es la media aritmética $\frac{1}{2 n} \sum_{b} \Delta I_{a b}$. La representación (6.6) es válida en este caso, hasta errores determinados por las diferencias medias de información $\Delta I_{a}$. Considérese entonces la representación aproximada:

$$
x_{a} y_{a}=\frac{M_{a}}{M} \varepsilon_{a}
$$

Para Fisher-Theil, $M=M$. ; $\varepsilon_{a}=\exp \frac{1}{2} \Delta I_{a}$. Por desgracia, es difícil obtener una expresión algebraica para $\varepsilon_{a}$ en el caso de los modelos con errores aditivos; éstos se verán empíricamente en la sección 7.

7. INGRESOS DEFLACIONADOS Y ESCALA DE CANTIDADES PARA OCHO REGIONES, México, 1963

Se han obtenido cantidades consumidas per capita durante un mes, para cada uno de los nueve agregados de alimentos y bebidas, y a partir de ellos se han calculado los cuatro índices de cantidades que se analizan en esta sección. El cuadro 9 consigna esta información primaria, que se obtuvo dividiendo las cantidades que aparecen en [1] entre la población de cada región,' según el censo de población de 1960. El último renglón de cada columna contiene el gasto mensual per capita a precios corrientes en alimentos, para cada región. Cabe notar que el consumo mensual per capita de alimentos en el país es de 112.74 pesos de 1963.

El cuadro 10 condensa las escalas para los modelos e índices considerados en este trabajo. Los resultados son semejantes a los que se refieren a los precios. La coincidencia total en cuanto a orden de las regiones, la coincidencia numérica muy cercana entre el modelo aditivo y el multiplicativo para el Fisher-Theil y el rango de variación relativamente pequeño del modelo multiplicativo aplicado a Paasche, Laspeyres y Fisher, características observadas para las escalas de precios, se observan en forma idéntica en las escalas de cantidades.

La relativa ineficiencia del modelo multiplicativo aplicado al Paasche, el Laspeyres y el Fisher y la eficiencia del multiplicativo para el Fisher-Theil y el aditivo para los demás índices, son inmediatamente apreciables; el lector puede calcular con facilidad estos resultados, que aquí se omiten. 
De nuevo, la posición de la región Golfo-Sureste llama la atención y contradice lo que tradicionalmente se cree respecto a esta zona. Sin embargo, se puede ver en el cuadro 9 que el consumo monetario de la región es bastante alto; si se ordenan las regiones en cuanto al gasto monetario per capita y se compara este orden con el de la escala de cantidades, sólo ocurren dos inversiones: la región Pacífico Norte y la Golfo-Sureste intercambian lugares (primero y segundo) y lo

\section{Cuadro 9}

México: Cantidades consumidas (EN Kilos) Y gasto MeNSUAL PER CAPITA PARA NUEVE AGREGADOS Y OCHO REGIONES, 1963

\begin{tabular}{|c|c|c|c|c|c|c|c|c|}
\hline dscggado Begión & 1 & 2 & 3 & 4 & 5 & 6 & 7 & 8 \\
\hline $\begin{array}{l}\text { 1. Halz y sus pro- } \\
\text { ductos }\end{array}$ & 5.2307 & 11.1864 & 16.2055 & 19.4176 & 12.0512 & 13.9826 & 6.3465 & 18.6503 \\
\hline $\begin{array}{l}\text { 2. Trigo y sus pro- } \\
\text { ductos }\end{array}$ & 7.0031 & 4.9070 & 4.1081 & 2.5528 & 2.4546 & 2.9389 & 5.2331 & 3.1363 \\
\hline $\begin{array}{l}\text { 3. Féoulas, leguni- } \\
\text { nosas }\end{array}$ & 7.2159 & 6.3897 & 6.2238 & 6.6544 & 4.1389 & 4.4716 & 3.8352 & 5.4027 \\
\hline 4. Azricares & 2.6924 & 2.5315 & 2.7564 & 2.6311 & 1.4226 & 1.8502 & 2.2003 & 2.7759 \\
\hline $\begin{array}{l}\text { 5. Verduras y fru- } \\
\text { tas }\end{array}$ & 7.1301 & 5.4225 & 8.4221 & 3.7816 & 3.5669 & 4.8698 & 9.1121 & 5.6514 \\
\hline 6. Gragas y aooites & $2 . \hat{8882}$ & 1.7720 & 1.4904 & 1.3130 & 0.9331 & 0.8247 & 1.3225 & 1.0237 \\
\hline $\begin{array}{l}\text { 7. Leohe y sus pro- } \\
\text { duoto: }\end{array}$ & 13.2551 & 9.2144 & 12.6718 & 8.7970 & 7.8857 & 8.0826 & 12.7890 & 6.9816 \\
\hline $\begin{array}{l}\text { 8. Carne, huevos } y \\
\text { marisoos }\end{array}$ & 4.9725 & 3.6574 & 5.3251 & 2.1525 & 1.6426 & 2.3541 & 4.4275 & 2.8879 \\
\hline 9. Otros produotos & 2.0043 & 1.2029 & 1.6580 & 1.0001 & 0.6140 & 0.9772 & 0.8825 & 2.2621 \\
\hline $\begin{array}{l}\text { Gasto mensial } \\
\text { percapita }\end{array}$ & 182.8714 & 137.1224 & 181.7373 & 106.2105 & 76.6799 & 90.1548 & 136.7751 & 111.3579 \\
\hline
\end{tabular}

Fuente: Referencia [1].

\section{Cuadro 10}

EsCaLAS CARDINALES aPROXIMADAS OPTIMAS DE CANTIDADES

\begin{tabular}{|c|c|c|c|c|c|c|c|c|c|}
\hline \multirow{4}{*}{ Región } & \multirow{4}{*}{$\begin{array}{c}\text { Nuimero } \\
\text { de } \\
\text { orden }\end{array}$} & \multicolumn{8}{|c|}{ Fecelas oandinales aproximadas óptimas } \\
\hline & & \multicolumn{2}{|c|}{ Wodelo multiplicativo } & \multicolumn{6}{|c|}{ Modelo aditivo } \\
\hline & & \multirow{2}{*}{$\begin{array}{l}\text { Parache, } \\
\text { Lagpeyres } \\
\text { y Blohox }\end{array}$} & \multirow{2}{*}{ Fishor-theil } & \multicolumn{3}{|c|}{ Magnitud restringtda } & \multicolumn{3}{|c|}{ Media restringida } \\
\hline & & & & Paasche & Laspegres & Figher & Pageche & Laspey ros & Piahor \\
\hline 1. Pacífico Joste & 2 & 1.0253 & 1.2293 & 1.1681 & 1.1922 & 1.1819 & 1.1914 & 1.2171 & 1.2053 \\
\hline 2. Morte & 4 & 1.0062 & 9.0798 & 1.0394 & 1.0335 & 1.0339 & 1.0557 & 1.0552 & 1.0550 \\
\hline 3. Golfo Sureste & 1 & 1.0410 & 1.3503 & 1.2954 & 1.2923 & 1.2934 & 1.3213 & 1.3194 & 1.3199 \\
\hline 4. Paclicico Contro & 6 & 0.9823 & 0.9160 & 0.8771 & 0.8726 & 0.8750 & 0.8948 & 0.8909 & 0.3930 \\
\hline 5. Bejro & 8 & 0.9402 & 0.6681 & 0.6421 & 0.6358 & 0.6393 & 0.6551 & 0.6493 & 0.6524 \\
\hline 6. Centro & 7 & 0.9683 & 0.8095 & 0.7784 & 0.7713 & 0.7750 & 0.7940 & 0.7875 & 0.7909 \\
\hline 7. Distrito Federal & 3 & 1.0117 & 1.1009 & 1.0655 & 1.0609 & 1.0630 & 1.0868 & 1.0833 & 1.0847 \\
\hline 8. Peoflico Sur & 5 & 0.9992 & 1.0236 & 0.9812 & 0.9767 & 0.9788 & 1.0008 & 0.9973 & 0.9988 \\
\hline
\end{tabular}


mismo hacen la región Norte y el Distrito Federal (tercero y cuarto). Estos cambios se explican totalmente en términos del nivel de precios. Se conjetura entonces que, si la creencia de que el nivel de consumo de la región es bajo resulta válida, la muestra del Banco de México está sesgada hacia los ingresos altos. Probablemente esto sólo podrá ser averiguado mediante nuevas encuestas de consumo.

$\mathrm{Si}$ bien ordinalmente los gastos monetarios y las escalas de cantidades manifiestan cierto parecido, cardinalmente las diferencias son mayores (ver cuadro 12, primeras dos columnas, que son escalas de gasto monetario relativo). De esto resalta la importancia de la descomposición del gasto monetario en el nivel de precios y el gasto real, aproximado este último por la escala cardinal de cantidades. Como se ha visto, esta descomposición sustituye en forma adecuada al concepto de "deflación", que por sí solo carece de sentido en vista de la incompatibilidad de los criterios de circularidad y reversión de factores.

El cuadro 11 presenta el resultado de aplicar la ecuación (6.5) a los índices de Fisher-Theil en las ocho regiones; se trata entonces del sesgo (multiplicativo) de reversión de factores del Fisher-Theil, que toma la forma de una diferencia de información entre estructuras del gasto familiar. Las unidades son nits o "unidades naturales de información" que resultan del uso de logaritmos naturales en la medición de la información.

Estos términos son relativamente pequeños: el mayor en valor absoluto es $\frac{1}{2} \Delta I_{48} \cong 0.0194$; el sesgo multiplicativo correspondiente es del orden del $2 \%$. Es de esperar entonces que el error al representar el ingreso monetario relativo por medio del producto de la escala de cantidades por la escala de precios sea también pequeño. El cuadro 12 tabula las siguientes relaciones:

$$
\varepsilon_{a}^{T}=\frac{x_{a} y_{a}}{M_{a} / M}
$$

Cuadro 11

DIFERENCIA DE INFORMACIÓN DE LAS DISTRIBUCIONES DEL GASTO FAMILIAR $1 / 2 \Delta I_{\mathrm{ab}}($ en nits)

\begin{tabular}{ccccccccc}
\hline b & 1 & 2 & 3 & 4 & 5 & 6 & 7 & 8 \\
\hline 1 & 0 & -.000947 & -.000843 & -.011254 & -.007974 & -.006301 & .000815 & .000351 \\
2 & .000947 & 0 & -.000159 & -.001465 & -.000734 & -.000296 & .002798 & .012967 \\
3 & .000843 & .000159 & 0 & .003032 & .002467 & .000822 & .001865 & .006224 \\
4 & .011254 & .001465 & -.003032 & 0 & .000198 & -.000061 & .016672 & .019410 \\
5 & .007974 & .000794 & -.002467 & -.000198 & 0 & .000052 & .013354 & .010530 \\
6 & .006301 & .000236 & -.000822 & .000061 & -.000052 & 0 & .011572 & .005176 \\
7 & -.000815 & -.002798 & -.001865 & -.016672 & -.013354 & -.011572 & 0 & -.012999 \\
8 & -.000351 & -.012967 & -.006224 & -.019410 & -.010530 & -.005176 & .012999 & 0 \\
\hline
\end{tabular}


en que $x_{a}, y_{a}$, son las escalas óptimas de precios y cantidades para el índice de Fisher-Theil y $\mathbf{M}$. es la media geométrica de ingreso monetario;

$$
\underset{\varepsilon_{a}}{\boldsymbol{p}}=\frac{x_{a} y_{a}}{M_{a} / \vec{M}}
$$

en que $x_{a}, y_{a}$ son las escalas para el índice de Paasche en el modelo aditivo con magnitud restringida y $\bar{M}$ es la media aritmética de ingreso monetario; en forma semejante se definen $\varepsilon_{a}^{L}$ y $\varepsilon_{a}^{p}$, para los índices de Laspeyres y Fisher, y:

$$
\delta_{a}^{P}=\frac{x_{a} y_{a}}{M_{a} / \bar{M}}
$$

en que $x_{a}, y_{a}$ son las escalas para el índice de Paasche en el modelo aditivo con media restringida; también se definen análogamente $\delta_{a}^{L}$ y $\delta_{a}^{F}$ para el Laspeyres y el Fisher.

\section{Cuadro 12}

\begin{tabular}{|c|c|c|c|c|c|c|c|c|c|}
\hline Región & $\frac{m_{a}}{M_{0}}$ & $\frac{n_{a}}{7}$ & $\epsilon_{\mathrm{a}}^{\mathrm{T}}$ & $\epsilon_{\mathrm{a}}^{\mathrm{P}}$ & $\epsilon_{\mathrm{a}}^{\mathbf{L}}$ & $\epsilon_{\mathrm{a}}^{\mathrm{F}}$ & $\delta_{a}^{P}$ & $\delta_{a}^{2}$ & $\delta_{a}^{P}$ \\
\hline 1. Pacifico Norto & 1.4919 & 1.4302 & .9982 & .9687 & 1.0101 & .9908 & .9926 & 1.0368 & 1.0164 \\
\hline 2. Norte & 1.1187 & 1.0724 & 1.0080 & .9903 & .9878 & .9885 & 1.0148 & 1.0142 & 1.0137 \\
\hline 3. Golfo Sureste & 1.4826 & 1.4213 & 1.0039 & .9936 & .9286 & .9902 & 1.0181 & 1.0145 & 1.0157 \\
\hline 4. Pacifico Centro & .8665 & .8307 & 1.0137 & .2954 & .9837 & .9897 & 1.0202 & 1.0099 & 1.0152 \\
\hline 5. Bafío & .6256 & .5997 & 1.0129 & .9975 & .9808 & .9399 & 1.0224 & 1.0072 & 1.0152 \\
\hline 6. Centro & .7355 & .7051 & 1.0107 & .993 & .0820 & .9204 & 1.0231 & 1.0082 & 1.0159 \\
\hline 7. Distrito FederaI & 1.1158 & 1.0697 & .9979 & .9939 & .9068 & .9900 & 1.0185 & 1.0132 & 1.0152 \\
\hline 8. Pacifico Sur & .9085 & .8709 & 1.0044 & .9961 & .9346 & .9899 & 1.0208 & 1.0109 & 1.0154 \\
\hline
\end{tabular}

ERRORES RELATIVOS EN LA REPRESENTACIÓN DEL INGRESO MONETARIO

Salvo dos casos excepcionales $\left(\varepsilon_{1}^{L}\right.$ y $\left.\delta_{1}^{P}\right)$ las escalas obtenidas a partir del modelo con errores aditivos sobrestiman o subestiman sistemáticamente el ingreso monetario relativo: sobreestiman cuando provienen de restringir la media de las escalas y subestiman cuando se restringe la magnitud. Sin embargo, estos errores son muy pequeños; en valor promedio son cercanos al $1 \%$; ver cuadro 13 . La escala de Kloek-Theil subestima en dos casos y sobrestima en los demás; su error medio es también muy pequeño $\left(1-\varepsilon_{a}^{T}\right)$ es, en la media, aproximadamente .0062 , o sea, menos del $1 \%$. 


\section{Cuadro 13}

VALORES MEDIOS DE $1-\varepsilon_{a}$ Y $1-\delta_{a}$

\begin{tabular}{cccr}
\hline \multicolumn{1}{c}{ Escala } & Paasche & Laspeyres & Fisher \\
\hline $\begin{array}{l}\text { Magnitud restringida }(\varepsilon) \\
\text { Media restringida }(\delta)\end{array}$ & -.0083 & -.0100 & -.0101 \\
\hline
\end{tabular}

Según esto, las escalas que más adecuadamente reproducen el gasto monetario relativo son la de Kloek-Theil y la del índice de Paasche con magnitud restringida. Esto, desde luego, es válido sólo para los datos analizados.

Por lo pronto, se puede pues concluir que la evidencia de esta muestra parece indicar que la descomposición del gasto monetario en un "componente real" y el nivel de precios es factible hasta errores bastante pequeños, y que esta descomposición sustituye con bastante éxito al concepto poco riguroso de "deflación" del gasto o ingreso monetario por medio de índices de precios.

\section{REFERENCIAS BIBLIOGRAFICAS}

[1] Banco de México, Oficina de Estudios sobre Proyecciones Agrícolas, Encuesta sobre ingresos y gastos familiares en México, 1963. México, Banco de México, Investigaciones Industriales, Oficina Editorial, 1966.

[2] Beckerman, W., International Comparisons of Real Per Capita Consumption Levels. OECD, Development Centre Studies N: 4, París, 1965.

[3] Bellman, R., Introduction to Matrix Analysis. Nueva York, McGraw Hill, 1961.

[4] Cseh-Szombathy, L., "Composing Synthetic Indicators for International Comparisons of the Standard of Living, Using Index Numbers Expressed in Natural Units of Measure", en The Standard of Living: Some Problems of Analysis and International Comparison. Budapest, Academia Húngara de Ciencias, 1962.

[5] Fisher, I., The Making of Index Numbers. Boston, Houghton-Miffin, 1922.

[6] Frisch R., "Necessary and Sufficient Conditions Regarding the Form of an Index Number Which Shall Meet Certain of Fisher's Tests", Journal of the American Statistical Association, 25 (1930), pp. 397-406.

[7] Gilbert, M. e I. Kravis, An International Comparison of National Products and the Purchasing Power of Currencies. París, OEEC, 1954.

[8] Kloek, T. y H. Theil, "International Comparisons of Prices and Quantities Consumed", Econometrica, 33 (1965), pp. 535-556.

[9] Kravis, I. y R. Lipsey, "The Use of Regression Methods in International Price Comparisons". Nueva York, National Bureau of Economic Research, noviembre de 1966, mimeografiado.

[10] Niewarowski, D. H., "The Level of Living of Nations: Meaning and Measurement". Banco Internacional de Reconstrucción y Fomento, Washington, julio de 1964, mimeografiado.

[11] Paige, D. y G. Bombach, A Comparison of National Output and Productivity of the United States and the United Kingdom, Paris, OEEC, 1959.

[12] Swamy, S., "Consistency of Fisher's Tests", Econometrica, 33 (1965), pp. 619-623.

[13] Theil, H., "The Information Approach to Demand Analysis", Econometrica, 33 (1965), pp. 67-87.

[14] Uribe, P., "Sobre la la agregación geométrica de precios y cantidades", docu- 
mento en preparación. Centro de Estudios Económicos y Demográficos, El Colegio de México.

[15] Van Ijzeren, J., "Three Methods of Comparing the Purchasing Power of Currencies", Statistical Studies, Núm. 7, Central Bureau voor de Statistiek, La Haya, 1956.

[16] Wald, A., "Zur Theorie der Preisindexziffern", Zeitschrift für Nationalökonomie, 8 (1937), pp. 179-219. 